\title{
An ethnobotanical survey of medicinal plants used in the East Sepik province of Papua New Guinea
}

Michael Koch', Dickson Andrew Kehop ${ }^{2}$, Boniface Kinminja², Malcolm Sabak², Graham Wavimbukie², Katherine M. Barrows ${ }^{3}$, Teatulohi K. Matainaho ${ }^{2}$, Louis R. Barrows ${ }^{3 *}$ and Prem P. Rai ${ }^{2}$

\begin{abstract}
Background: Rapid modernization in the East Sepik (ES) Province of Papua New Guinea (PNG) is resulting in a decrease in individuals knowledgeable in medicinal plant use. Here we report a synthesis and comparison of traditional medicinal plant use from four ethnically distinct locations in the ES Province and furthermore compare them to two other previous reports of traditional plant use from different provinces of PNG.

Methods: This manuscript is based on an annotated combination of four Traditional Medicines (TM) survey reports generated by University of Papua New Guinea (UPNG) trainees. The surveys utilized a questionnaire titled "Information sheet on traditional herbal preparations and medicinal plants of PNG", administered in the context of the TM survey project which is supported by WHO, US NIH and PNG governmental health care initiatives and funding. Regional and transregional comparison of medicinal plant utilization was facilitated by using existing plant databases: the UPNG TM Database and the PNG Plant Database (PNG Plants) using Bayesian statistical analysis.

Results: Medicinal plant use between four distinct dialect study areas in the ES Province of PNG showed that only a small fraction of plants had shared use in each area, however usually utilizing different plant parts, being prepared differently and to treat different medical conditions. Several instances of previously unreported medicinal plants could be located. Medicinally under- and over-utilized plants were found both in the regional reports and in a transregional analysis, thus showing that these medicinal utilization frequencies differ between provinces.

Conclusions: Documentation of consistent plant use argues for efficacy and is particularly important since established and effective herbal medicinal interventions are sorely needed in the rural areas of PNG, and unfortunately clinical validation for the same is often lacking. Despite the existence of a large corpus of medical annotation of plants for PNG, previously unknown medical uses of plants can be uncovered. Furthermore, comparisons of medicinal plant utilization is possible if databases are reformatted for consistencies that allow comparisons. A concerted effort in building easily comparable databases could dramatically facilitate ethnopharmacological analysis of the existing plant diversity.
\end{abstract}

Keywords: Papua New Guinea, East Sepik, Medicinal plants, Bougainville, Eastern highlands, Quantitative ethnopharmacology

\footnotetext{
*Correspondence: Ibarrows@pharm.utah.edu

Deceased

${ }^{3}$ Department of Pharmacology and Toxicology, University of Utah, 30 S. 2000

E., Salt Lake City 84112 UT, USA

Full list of author information is available at the end of the article
}

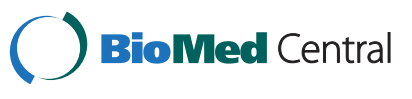

(c) 2015 Koch et al. Open Access This article is distributed under the terms of the Creative Commons Attribution 4.0 International License (http://creativecommons.org/licenses/by/4.0/, which permits unrestricted use, distribution, and reproduction in any medium, provided you give appropriate credit to the original author(s) and the source, provide a link to the Creative Commons license, and indicate if changes were made. The Creative Commons Public Domain Dedication waiver (http://creativecommons.org/publicdomain/zero/1.0/) applies to the data made available in this article, unless otherwise stated. 


\section{Background}

Papua New Guinea (PNG) is a largely rural country characterized by at least 800 ethnic traditions dispersed over $462,840 \mathrm{~km}^{2}$ [1,2]. Most of the population resides in small villages, situated in diverse environs that range from montane rainforest to lowland river deltas and small tropical islands. Settled 49,000-44,000 years ago (Ivane Valley in the PNG Highlands) [3], PNG is blessed with extraordinary biological diversity and a rich but fragmented cultural tapestry of customs, art, spiritual beliefs and medicinal knowledge.

The East Sepik Province is situated in the northwest of the country bordered by the West Sepik Province (West), Madang Province (East), the Bismark Sea (North) and Enga Province (South). East Sepik $\left(43,426 \mathrm{~km}^{2}\right)$ is characterized by mountainous terrain to the south and west and the costal floodplain of the Sepik river, which flows west to east through the province [4]. The approximately 350,000 inhabitants have to rely on 37 health centers for provisioning health care and heavily supplement western medicines with traditional medicines (TM) $[4,5]$. The $10 \%$ mortality rate for children under 5 years reflects the difficulty of providing adequate health care in the East Sepik Province. In an effort to supplement health care with effective traditional medicins the University of Papua New Guinea (UPNG), endorsed by the PNG government, struck a collaboration with the WHO to develop reliable traditional medicines (TM) and safe practices (outlined in the 2001-2010 PNG National Health Plan [6]). Part of this project includes traditional medicine surveys performed by UPNG students working in their kinship ("wantok") communities. The data are then recorded in a proprietary database maintained at UPNG [7]. This database serves as central repository for PNG traditional medicine practices, preserving cultural traditions from many diverse communities.

\section{Methods}

The TM surveys are performed by UPNG students who are instructed on plant identification, preservation, herbal medicine use, and trained on how to administer the survey instrument entitled "Information sheet on traditional herbal preparations and medicinal plants of Papua New Guinea." The survey questionnaire is the basis for semistructured face-to-face interviews with healers, herbalists, birth attendants, and bone setters. Field vouchers of medicinal plants (twigs with leaves, fruits, flowers, nuts, etc.) are harvested under guidance of the healer and dried and compressed in newspapers. Photographs, descriptions and the pressed plant samples are assigned a voucher number and deposited with the UPNG Herbarium for later identification and reference [8].

The data concerning plant use are written up under supervision into student authored reports and the plant information is entered into the UPNG Traditional Medicines Database, which contains the combined data from reports generated by a decade's work in this endeavor. It is the student reports that provide the base information for this current report.

Four student reports from the East Sepik Province representing four distinct language dialect communities have been compiled here: "Traditional Medicinal Plants and Practices in the Waskuk Hills Area of Ambunti District in East Sepik (2005)" by Dickson Andrew Kehop; "Traditional Medicine Practices in Niungweko and Kunjingini (MUL) Area of Wosera-Gawi District in East Sepik (2006)" by Boniface Kinminja; "Enthnobotanical Survey of Traditional Medicine in East Yangoru, East Sepik Province, Papua New Guinea" (2004) by Graham Wavimbukie; and "Traditional Medicinal Plants and Practices in Kairiru Island East Sepik Province Papua New Guinea (2004) by Malcolm Sabak The first three reports (DK, BK and GW, respectively) are inland above the Sepik floodplain at elevations ranging from 150 to $300 \mathrm{~m}$. The report from Kairiru Island is referred to as MS. The specific village communities interviewed included: Bangus and Mariawai villages (DK), Niungweko and Mul (Kunjingini 1) villages (BK); Marambanja, Saina, Ambukanja, Parina, Jawia, Mandien, Bukiendoun, Sausenduon, Hangrerak and Kiarivu villages (GW) and Rumlal, Shagur and Bou villages (MS).

A compilation of references for medicinal plants described from Papua New Guinea is currently in progress (50 references) in our lab. These references were used to determine if plants collected in the current East Sepik survey work have been previously reported for medicinal use. Comparison of this East Sepik medicinal plant report to our previous reports from Bougainville [8] and the Eastern Highlands [9] was accomplished after editing the previous two reports to match the current format, including codes for conditions treated.

Overall flora distribution data for PNG was obtained for the provinces of East Sepik, Eastern Highlands, and the autonomous region of Bougainville from the PNG Plants Database [10]. The data was imported into Microsoft Excel $^{\mathrm{m}}$, formatted and edited as necessary, then processed with standard Unix (Linux) utilities to produce a formatted list containing the plant family, genus and species (if known). Duplicated instances of plants in the list were removed. The family names were split off, sorted and processed using a Python script on a Raspberry Pi Model B (http://www.raspberrypi.org) to quantify the number of instances of each plant family cited in the list. In general, when multiple names for the same species were found, we attempted to match plant family names to the PNG Plant Database versions to allow for quantitative comparison. Statistical analysis was carried out exactly as previously described by Weckerle et al. [11] using the 
"beta.inv" function provided in Microsoft Excel ${ }^{\mathrm{TM}}$ to calculate the $95 \%$ inferior and superior credible intervals for the data. Comparison of the currently reported East Sepik medical plants to the Traditional Medicines Plant Database maintained at UPNG was carried out similarly. Discrepancies in plant family names were resolved as described above, the family names were adapted to the family names in the UPNG Traditional Medicines Database; resulting in a slightly different number of plant families for the traditional plant uses reported.

Plant families which are considered overused have an inferior credible interval that lies above the superior credible interval for the regional total data. Plant families that are considerend underused have a superior credible interval below the inferior credible interval of the regional total data set distribution.

\section{Results and discussion \\ East Sepik reports}

The combined student reports contained 299 entries (including unidentified plants $(n=6)$ ) from the East Sepik province of PNG. The reports collated 205 plants, of which 139 were identified to species and 66 to genus, from a total of 71 families. Three reports were from areas of estimated $150 \mathrm{~m}$ to $300 \mathrm{~m}$ elevation (DK, BK and GW voucher numbers), DK from Waskuk Hills in the center of Sepik province, and BK and GW from elevated areas of the province north of the Sepik river plain and southwest of the capital Wewak. One report (MS voucher numbers) came from an island (Kairiru Island) situated close to the coast of East Sepik. The combined dataset is presented in Table 1 for all four areas of the East Sepik province under consideration.

\section{Shared and unique plants}

We found a number of plants were reported as used in common amongst these areas. Many plants had many overlaps in use, preparation, and disease (Table 1). However, among the plants identified to species level, only four species were reported in every survey: Alstonia scholaris (L.) R.Br., Cassia alata L., Passiflora foetida L., and Zingiber officianale Roscoe. The number of plants unique to one or another of the four reports was surprisingly large in comparison to the previous reports [8, 9]. A total of 80 genera, of which 29 are identified to genus level and 51 to species level (see Table 2), were not shared between any of the four study areas.

\section{Plant parts utilization, preparation, administration and diseases treated}

In general the areas studied were similar in the relative utilization of plant parts (Fig. 1) with leaves predominating followed by bark and sap as next most common (with the exception of GW where roots were more commonly utilized than sap). The MS sample set reported a large number of young shoots/young roots stipulated for use in comparison to the other reports, where "young" was not specifically stipulated. The DK and GW reports only cited use of shoots. Only DK reported the medicinal use of nuts.

The method of preparation (Fig. 2) shows a similar pattern amongst the reports: use of succus (expressed juice) was most commonly reported, followed by decoction and direct application of the raw plant material. Usually direct application meant placing the material on a wound or skin ailment after minimal handling. Similarly all reports contain inhalation of smoke or vapor, heat treatment and cooking prior to utilization. DK reported a much higher frequency of cooking the material than the other areas. Boiling as a method of preparation was only mentioned in the MS and GW reports, while mastication (chewing) was reported in all except MS. Typically heating implies later consumption or preparation of steam for inhalation, however, in the GW report heating is a method to prepare the plant material prior to topical application (labelled HR-HeatedRubbed). Another mode of preparation was mastication and spitting on the affected area. This was relatively common in the MS report and mentioned in the DK report, but not noted in the the other two areas. Only from the DK report is the reverse utilization of the plants reported, where in one instance Homalium foetidum (Roxb.) Benth. was utilized in a reverse-fromexpected manner. In this case, the blood of the patient was placed under the bark of the tree with the expected result being a lessening of knee pain and strengthening of bones as the tree grew. This clearly implies a spiritual/ magical connection of plant and patient.

The routes of administration for plant based medicines reported by DK, BK and MS were about evenly divided between oral or topical routes (Fig. 3). The exception was the administration practices reported by GW where oral consumption outpaced topical application (3:2 ratio). Inhalation was reported only once for the DK and $\mathrm{BK}$ areas, and more frequently in the BW and MS areas. The lone outlier for route of administration was from the DK report in which patient material (blood) was transferred to the plant (as described above).

Ailments treated (Fig. 4) with plant based medicines were categorized into 28 groups, sorted according to the target site, in order to to minimize possibly uncertain medical judgements or clinical misdiagnoses. Many described symptoms can likely accurately be ascribed to their appropriate causative diseases, but in the absence of independent clinical confirmation the decision was made to present the data in as unbiased a way as possible. Therefore, the category of "SKIN" contains both 
Table 1 Plants reported as medicinally used in 4 study areas in East Sepik Province

\begin{tabular}{|c|c|c|c|c|c|c|c|c|}
\hline Voucher & Plant ID References & Family & Local Name & Ailment & AilmentCode & PartCode & PrepCode & RouteCode \\
\hline MS 02/04 & Abelmoschus manihot (L.) Medik [32-39] & Malvaceae & Wasniat & Uterine contraction & REP & L & $\mathrm{D}$ & $\mathrm{O}$ \\
\hline GW 05/04 & Acalypha grandis Benth $[33,40]$ & Euphorbiaceae & Unknown & $\begin{array}{l}\text { Antidote to poisoning } \\
\text { (Chemical or acid) }\end{array}$ & POIS & L & S & $\mathrm{O}$ \\
\hline DK 16/05 & Acalypha sp. [7, 33-35, 39-49] & Euphorbiaceae & Mikirme & Malaria & MAL & L & $\mathrm{D}$ & $\mathrm{O}$ \\
\hline GW 88/04 & Acalypha sp. [7, 33-35, 39-49] & Euphorbiaceae & Winghongong & Cough, shortness of breath & RESP & Sap & s & $\mathrm{O}$ \\
\hline BK 057/06 & $\begin{array}{l}\text { Acalypha wilkesiana Müll. Arg } \\
{[42,44,45,47-49]}\end{array}$ & Euphorbiaceae & Polembieri & Cough, shortness of Breath & RESP & L & $\mathrm{D}$ & O \\
\hline DK 38/05 & Ageratum conyzoides (L.) L [8] & Asteraceae & Mungrimb & Sore & SKIN & L & $\mathrm{R}$ & T \\
\hline GW 56/04 & Aglaia sp. $[33,50,51]$ & Meliaceae & Waniembri & Fevers, malaria & FEV/MAL & L & B & । \\
\hline GW 09/04 & Albizia procera (Roxb.) Benth [34] & Fabaceae & He're & Malaria, pneumonia, asthma & MAL/RESP & B & S & $1 \& O$ \\
\hline BK 058/06 & Albizia saman (Jacq.) Merr [34, 52] & Mimosaceae & Yundimi & Induce sleep & PSYCH & L & $\mathrm{D}$ & T \\
\hline DK 08/05 & Allophylus cobbe (L.) Raeusch $[8,42,51,53]$ & Sapindaceae & Haim & Scabies & SKIN & B & C & $\mathrm{O}$ \\
\hline GW 50/04 & Allophylus cobbe (L.) Raeusch $[8,42,51,53]$ & Sapindaceae & Wah & Skin pox, cough & SKIN/RESP & L & $\mathrm{D}$ & $\mathrm{T} \mid \mathrm{O}$ \\
\hline DK $37 / 05$ & Alocasia cucullata (Lour.) G. Don & Araceae & Waken & Boil & SKIN & Root & $\mathrm{R}$ & $\mathrm{T}$ \\
\hline MS 07/04 & Alocasia sp. $[8,33-35,43,50,51]$ & Araceae & Waiyat & Abortion & REP & L & S & $\mathrm{O}$ \\
\hline GW 27/04 & $\begin{array}{l}\text { Alphitonia incana (Roxb.) Teijsm. \& } \\
\text { Binn. ex Kurz }[33-35,43,54]\end{array}$ & Rhamnaceae & Hushu & Scabies & SKIN & B & S & T \\
\hline GW 24/04 & Alpinia sp. $[8,9,33,34,42,43,55-57]$ & Zingiberaceae & Wambelekie & Cancer (mouth), hypertension & CANC/CV & $\mathrm{R}$ & $\mathrm{D}$ & $\mathrm{O}$ \\
\hline MS 03/04 & Alpinia sp. $[8,9,33,34,42,43,55-57]$ & Zingiberaceae & Kasai & Cough & RESP & yShoot & S & $\mathrm{O}$ \\
\hline MS 41/04 & Alpinia sp. $[8,9,33,34,42,43,55-57]$ & Zingiberaceae & Sinup & Fever, headache, body ache & $\begin{array}{l}\text { FEV/HEAD/PAIN/ } \\
\text { SWELL }\end{array}$ & yShoot & S & $\mathrm{O}$ \\
\hline MS 68/04 & Alpinia sp. $[8,9,33,34,42,43,55-57]$ & Zingiberaceae & Kasai & Antidepressant & PSYCH & L \& yShoot & s & $\mathrm{O}$ \\
\hline BK 022/06 & $\begin{array}{l}\text { Alstonia scholaris (L.) R.Br } \\
{[7-9,34,39,40,42,43,46-49,51-53,57-64]}\end{array}$ & Apocynaceae & Kam-bh & Malaria, diarrhoea, asthma, sores & $\begin{array}{l}\text { MAL/GAST/RESP/ } \\
\text { SKIN }\end{array}$ & $\begin{array}{l}\text { L } \mid \text { Sap } \mid \\
\text { Sap }\end{array}$ & $D|D| S$ & $\mathrm{O}$ \\
\hline DK 25/05 & $\begin{array}{l}\text { Alstonia scholaris (L.) R.Br } \\
{[7-9,34,39,40,42,43,46-49,51-53,57-64]}\end{array}$ & Apocynaceae & Chimb & Scabies & SKIN & B & C & $\mathrm{O}$ \\
\hline GW 16/04 & $\begin{array}{l}\text { Alstonia scholaris (L.) R.Br }[7-9,34,39,40,42,43, \\
46-49,51-53,57-64]\end{array}$ & Apocynaceae & Hembe & Fever, malaria, cough, diarrhoea & $\begin{array}{l}\text { FEV/MAL/RESP/ } \\
\text { GAST }\end{array}$ & Sap & S & $\mathrm{O}$ \\
\hline MS 04/04 & $\begin{array}{l}\text { Alstonia scholaris (L.) R.Br }[7-9,34,39,40,42,43 \text {, } \\
46-49,51-53,57-64]\end{array}$ & Apocynaceae & Kaisabok & Fever, headache & FEV/HEAD & B & $\mathrm{D}$ & $\mathrm{O}$ \\
\hline BK 034/06 & Amomum aculeatum Roxb $[39,42,53,58,65]$ & Zingiberaceae & Takkwa hamba & Asthma, scabies & RESP/SKIN & Stem & C & $O \& T$ \\
\hline DK 19/05 & Amomum aculeatum Roxb $[39,42,53,58,65]$ & Zingiberaceae & Guinj Nikir & Fever & FEV & Whole & V & । \\
\hline DK 53/05 & Angiopteris evecta (G. Forst.) Hoffm $[8,56]$ & Marattiaceae & Yarchapa & Shortness of breath & RESP & $\begin{array}{l}\text { Shoot \& } \\
\text { Root }\end{array}$ & S & $\mathrm{O}$ \\
\hline MS 01/04 & Archidendron sp. $[8,66]$ & Fabaceae & Niar & Diarrhoea, asthma, fever, headache & HEAD/FEV/GAST & B & $\mathrm{D}$ & 0 \\
\hline
\end{tabular}


Table 1 Plants reported as medicinally used in 4 study areas in East Sepik Province (Continued)

\begin{tabular}{|c|c|c|c|c|c|c|c|c|}
\hline DK 02/05 & Areca catechu L $[8,34,37,39,42,43,51,67,68]$ & Arecaceae & Maimb & Abdominal ache, whitespots & GAST/SKIN & $\begin{array}{l}\text { Shoot } \\
\text { Nut }\end{array}$ & $R \mid R$ & $\mathrm{~T} \mid \mathrm{O}$ \\
\hline MS 10/04 & Aristolochia sp. $[8,9,34,43,48,56,59,61,63]$ & Aristolochiaceae & Mutamuth & Epigastric pain & GAST & L & $\mathrm{R}$ & T \\
\hline MS 73/04 & Aristolochia sp. $[8,9,34,43,48,56,59,61,63]$ & Aristolochiaceae & War sapiau & Blocked nose, flu, cough & RESP & L & $\mathrm{R}$ & I \\
\hline MS 23/04 & $\begin{array}{l}\text { Artocarpus altilis (Parkinson ex F.A. Zorn) } \\
\text { Fosberg }[8,34,43,50,61]\end{array}$ & Moraceae & Kaikning & Hemorrhage & WOUND & Sap & $\mathrm{R}$ & $\mathrm{O}$ \\
\hline GW 79/04 & Asclepias sp. & Apocynaceae & Huaraloho & Enlarged spleen & ORG & Root & S & $\mathrm{O}$ \\
\hline DK 21/05 & Asplenium nidus $L[8,53]$ & Aspleniaceae & Yimangir & Infant back ache & CHILD & L & $\mathrm{R}$ & $\mathrm{T}$ \\
\hline BK 039/06 & Averrhoa carambola L. & Oxalidaceae & Macosembi & Aasthma, sore, fresh cut & $\begin{array}{l}\text { RESP/SKIN/ } \\
\text { WOUND }\end{array}$ & Fruit & $R \mid R$ & $\mathrm{O} \mid \mathrm{T}$ \\
\hline DK 01/05 & Averrhoa carambola L. & Oxalidaceae & Waskapui & Cough & RESP & Fruit & $\mathrm{D}$ & $\mathrm{O}$ \\
\hline MS 27/04 & $\begin{array}{l}\text { Barringtonia asiatica (L.) Kurz } \\
{[38,39,42,46,51,59,64]}\end{array}$ & Lecythidaceae & Wut & Antipsychotic & PSYCH & B & $\mathrm{D}$ & $\mathrm{O}$ \\
\hline GW 40/04 & $\begin{array}{l}\text { Bidens pilosa } \mathrm{L} \text {. } \\
{[7,9,32,34-36,39,42,43,50,57,60]}\end{array}$ & Asteraceae & Miniesihaik & Eye infections, bleeding & INF/WOUND & Root & s & T \\
\hline DK 11/05 & Bixa orellana $\mathrm{L}[7,35,42,43,50,53]$ & Bixaceae & Noksinu & Grille & SKIN & Seed & s & T \\
\hline MS 53/04 & $\begin{array}{l}\text { Breynia sp. } \\
{[7,33,34,39,42,43,50,53,58,68,69]}\end{array}$ & Phyllanthaceae & Smallak & Sore gums & DENT & yShoot & S & $\mathrm{T}$ \\
\hline MS 61/04 & $\begin{array}{l}\text { Breynia sp. } \\
{[7,33,34,39,42,43,50,53,58,68,69]}\end{array}$ & Phyllanthaceae & Murpopau & Fever, joint pain, headache (severe) & FEV/PAIN/HEAD & B & s & $\mathrm{O}$ \\
\hline GW 21/04 & $\begin{array}{l}\text { Bryophyllum pinnatum (Lam.) Oken } \\
{[7,42,51,67]}\end{array}$ & Crassulaceae & Golip & Strong cough & RESP & L & $\mathrm{D}$ & $\mathrm{O}$ \\
\hline MS 21/04 & $\begin{array}{l}\text { Bryophyllum pinnatum (Lam.) Oken } \\
{[7,42,51,67]}\end{array}$ & Crassulaceae & Mitultul & Ulcer & SKIN & L & $\mathrm{H}$ & $\mathrm{T}$ \\
\hline BK 006/06 & Calamus sp. $[8,33,35,43,53,57]$ & Arecaceae & Bal & $\begin{array}{l}\text { Fever, headache, malaria, cough, } \\
\text { malnutrition }\end{array}$ & $\begin{array}{l}\text { FEV/HEAD/MAL/ } \\
\text { NUT }\end{array}$ & Sap & s & $\mathrm{O}$ \\
\hline BK 051/06 & Calamus sp. $[8,33,35,43,53,70]$ & Arecaceae & Gwalkipi & Dehydration & NUT & Sap & s & $\mathrm{O}$ \\
\hline GW 92/04 & Calamus sp. $[8,33,35,43,53,64]$ & Arecaceae & Peli & General cleansing & MAINT & Sap & S & $\mathrm{O}$ \\
\hline MS 38/04 & Calamus sp. $[8,33,35,43,53,64]$ & Arecaceae & War huk & Asthma & RESP & Sap & S & $\mathrm{O}$ \\
\hline MS 85/04 & Callicarpa longifolia Lam $[34,51]$ & Verbenaceae & Yeaik & Sore in baby's mouth & CHILD & B & MS & $\mathrm{T}$ \\
\hline MS 20/04 & $\begin{array}{l}\text { Calophyllum inophyllum } L . \\
{[34,39,42,43,61,67]}\end{array}$ & Guttiferae & Sabour & Toothache & DENT & B & $\mathrm{D}$ & $\mathrm{O}$ \\
\hline MS 32/04 & Calotropis gigantea (L.) (L.) Dryand [51] & Apocynaceae & Sasus & Fever, headache & FEV/HEAD & L & V & । \\
\hline DK 56/05 & Campnosperma brevipetiolatum Volkens [71] & Anacardiaceae & Gwart & Ulcer & SKIN & Sap & S & $\mathrm{T}$ \\
\hline BK 010/06 & Campnosperma sp. & Anacardiaceae & Biakuar & $\begin{array}{l}\text { Sores, scabies, fresh cut, wound, } \\
\text { hair and skin (as oil), removal of } \\
\text { spear in skin }\end{array}$ & WOUND/SKIN & B & s & T \\
\hline
\end{tabular}


Table 1 Plants reported as medicinally used in 4 study areas in East Sepik Province (Continued)

\begin{tabular}{|c|c|c|c|c|c|c|c|c|}
\hline MS 39/04 & Canarium sp. $[34,42,43,50,57,70]$ & Burseraceae & Klakul & Emetic & GAST & B & S & O \\
\hline MS 64/04 & Canarium sp. $[34,42,43,50,57,70]$ & Burseraceae & Yamuok & Ulcer & SKIN & Sap & S & T \\
\hline DK 15/05 & Capsicum annuum L. & Solanaceae & Seraimbsik & Malaria & MAL & $\begin{array}{l}\text { Fruit \& } \\
\text { Seed }\end{array}$ & C & $\mathrm{O}$ \\
\hline DK 34/05 & Carica papaya $\mathrm{L}[42,43,46,47,59,64,68]$ & Caricaceae & Pous & Malaria & MAL & Root & $\mathrm{D}$ & $\mathrm{O}$ \\
\hline DK 26/05 & Caryota mitis Lour. & Arecaceae & Tosh & Shortness of Breath & RESP & Succus & S & $\mathrm{O}$ \\
\hline MS 69/04 & Caryota rumphiana Mart. $[39,53]$ & Arecaceae & Yamoun & Toothache & DENT & yShoot & M & $\mathrm{O}$ \\
\hline BK 028/06 & Cascabela thevetia (L.) Lippold [51] & Apocynaceae & Lai & Sores & SKIN & L \& Seed & s & T \\
\hline BK 044/06 & $\begin{array}{l}\text { Cassia alata } \mathrm{L} \\
{[7,8,34,37,38,42,46,49,52,59,61,63,67,69]}\end{array}$ & Fabaceae & Yundilipgi & Grille and white spot & SKIN & L & $\mathrm{R}$ & T \\
\hline DK 43/05 & $\begin{array}{l}\text { Cassia alata } \mathrm{L} \\
{[7,8,34,37,38,42,46,49,52,59,61,63,67,69]}\end{array}$ & Fabaceae & Apkuaiamboi & Grille & SKIN & L & $H \mid R$ & T \\
\hline GW 01/04 & $\begin{array}{l}\text { Cassia alata } \mathrm{L} \\
{[7,8,34,37,38,42,46,49,52,59,61,63,67,69]}\end{array}$ & Fabaceae & Kenjimbi & $\begin{array}{l}\text { Fungal infections, tinea, } \\
\text { (white spot, grille }\end{array}$ & INF/SKIN & L & $H \mid R$ & $\mathrm{~T}$ \\
\hline MS 74/04 & $\begin{array}{l}\text { Cassia alata L }[7,8,34,37,38,42,46,49,52,59,61 \text {, } \\
63,67,69]\end{array}$ & Fabaceae & Piaktie & Ggrille & SKIN & L & $\mathrm{H}$ & T \\
\hline GW 71/04 & Cassia sp. & Fabaceae & Pipi & Female infertility & REP & Root & $\mathrm{D}$ & $\mathrm{O}$ \\
\hline MS 28/04 & $\begin{array}{l}\text { Casuarina equisetifolia } L \\
{[33,34,38-40,42,43,46,58,72]}\end{array}$ & Casuarinaceae & Kaiklee & Scabies, skin pox, small sores & SKIN & B & $\mathrm{D}$ & T \\
\hline MS 19/04 & Cenchrus sp. & Gramineae & Warawara & Cough & RESP & Stem & M & O \\
\hline GW 94/04 & Cenchrus sp. & Gramineae & Mitate & Enlarged spleen & ORG & L & D & O \\
\hline GW 12/04 & Cerbera floribunda K. Schum [51] & Apocynaceae & Yaung & Malaria, pneumonia & MAL/RESP & B & $\mathrm{D}$ & $\mathrm{O}$ \\
\hline DK 20/05 & $\begin{array}{l}\text { Cheilocostus speciosus (J. König) C. Specht } \\
{[8,34,42,47,56]}\end{array}$ & Costaceae & Yangir & Shortness of Breath & RESP & Succus & s & O \\
\hline BK 008/06 & Christia sp. & Fabaceae & Banjip & $\begin{array}{l}\text { Diarrhoea, scabies, sores on the } \\
\text { head like scabies }\end{array}$ & GAST/SKIN & L & $D \mid R$ & $\mathrm{O} \mid \mathrm{T}$ \\
\hline MS 50/04 & Chrysopogon aciculatus (Retz). Trin & Poaceae & Knarbru & Swollen bodies, legs, arms & SWELL & Whole & D & T \\
\hline DK 54/05 & Cinnamonum sp. & Lauraceae & Metamboi & Headache & HEAD & B & MS & T \\
\hline GW 59/04 & Cissus sp. $[33,34,43,53,59,62]$ & Vitaceae & Lenghasa & Stomach ache, diarrhoea & GAST & Sap & s & O \\
\hline BK 049/06 & $\begin{array}{l}\text { Clematis sp. } \\
{[8,33,34,37,39,42,43,51,53,59,65-67,69]}\end{array}$ & Ranunculaceae & Gwawingga & Nasal congestion, running nose & RESP & L & V & I \\
\hline GW 87/04 & Clerodendrum sp. $[8,37,38,62]$ & Labiatae & Hambaihile & Snake bite & BITE & Sap & s & $\mathrm{O}$ \\
\hline GW 91/04 & Clitoria ternatea $\mathrm{L}$. & Fabaceae & Pohuk & $\begin{array}{l}\text { Determine female sex for } \\
\text { baby, infertility }\end{array}$ & REP & Fruit & C & $\mathrm{O}$ \\
\hline MS 78/04 & $\begin{array}{l}\text { Cocos nucifera } L \\
{[7,8,34,37-39,43,56,59,61,67,68]}\end{array}$ & Arecaceae & Niumour & Bleeding from cuts & WOUND & Fruit & $\mathrm{H}$ & T \\
\hline
\end{tabular}


Table 1 Plants reported as medicinally used in 4 study areas in East Sepik Province (Continued)

\begin{tabular}{|c|c|c|c|c|c|c|c|c|}
\hline BK 047/06 & $\begin{array}{l}\text { Codiaeum variegatum (L.) Rumph. ex A. Juss } \\
{[8,34,35,39,42,43,48,56,58,61,62,66,73]}\end{array}$ & Euphorbiaceae & Diripmi & Ulcer & SKIN & Sap & $S$ & $\mathrm{~T}$ \\
\hline MS 37/04 & $\begin{array}{l}\text { Codiaeum variegatum (L.) Rumph. ex A. Juss } \\
{[8,34,35,39,42,43,48,56,58,61,62,66,73]}\end{array}$ & Euphorbiaceae & Waeke & Ssores around the mouth area & SKIN & Succus & S & T \\
\hline DK $12 / 05$ & $\begin{array}{l}\text { Cordyline fruticosa (L.) A. Chev } \\
{[37,38,45,56,59-61]}\end{array}$ & Asparagaceae & Awa & Grille & SKIN & B \& Stem & R & T \\
\hline GW 86/04 & $\begin{array}{l}\text { Cordyline fruticosa (L.) A. Chev } \\
{[37,38,45,56,59-61]}\end{array}$ & Asparagaceae & Haua & Fresh cuts, sores & WOUND/SKIN & $\mathrm{L}$ & $\mathrm{H}$ & T \\
\hline MS $67 / 04$ & $\begin{array}{l}\text { Cordyline fruticosa (L.) A. Chev } \\
{[37,38,45,56,59-61]}\end{array}$ & Asparagaceae & Shir & Fever, headache, general body pain & FEV/HEAD/PAIN & $\begin{array}{l}L \& \\
\text { yShoot }\end{array}$ & S & $\mathrm{O}$ \\
\hline BK 053/06 & $\begin{array}{l}\text { Crinum asiaticum } \mathrm{L} \\
{[7,8,34,39,42,49,51,56,61,62,67,74]}\end{array}$ & Amaryllidaceae & Yawal & Swollen leg, limbs, muscles & SWELL & $\mathrm{L}$ & $\mathrm{H}$ & T \\
\hline GW 39/04 & $\begin{array}{l}\text { Crinum asiaticum } L \\
{[7,8,34,39,42,49,51,56,61,62,67,74]}\end{array}$ & Amaryllidaceae & Youri & General cleansing, swollen breast & GAST/SWELL & Sap \& L & $\mathrm{S} \mid \mathrm{HR}$ & $\mathrm{O} \mid \mathrm{T}$ \\
\hline MS 29/04 & $\begin{array}{l}\text { Crinum asiaticum } \mathrm{L} \\
{[7,8,34,39,42,49,51,56,61,62,67,74]}\end{array}$ & Amaryllidaceae & Milakiap & Scabies, rectal prolapse & SKIN/GAST & Stem & $S$ & $\mathrm{O} \mid \mathrm{T}$ \\
\hline MS 54/04 & $\begin{array}{l}\text { Crinum asiaticum var. asiaticum } \\
{[34,54,61]}\end{array}$ & Amaryllidaceae & Kalava & Anemia & BLOOD & L & D & $\mathrm{O}$ \\
\hline GW 75/04 & Cryptocarya sp. $[8,33-35,43,50,53,55,65]$ & Lauraceae & Misipi (misi-ph) & Cough, clear thinking & RESP/PSYCH & B & $\mathrm{D}$ & $\mathrm{O}$ \\
\hline BK 035/06 & Cryptocarya sp. $[8,33-35,43,50,53,55,65]$ & Lauraceae & Kovi & Malaria and stomach ache & MAL/GAST & B & $\mathrm{R}$ & $\mathrm{O}$ \\
\hline BK 029/06 & Curcuma longa $L[34,42,45]$ & Zingiberaceae & Laki & Poison by black magic & MAGIC & Root & $\mathrm{R}$ & $\mathrm{O}$ \\
\hline GW 35/04 & Curcuma sp. $[34,35,40,42-45,62]$ & Zingiberaceae & Hivinguambe & Fever, headache & FEV/HEAD & Shoot & B & । \\
\hline GW 38/04 & Curcuma sp. $[34,35,40,42-45,62]$ & Zingiberaceae & Lekienga & Broken bones, curds/boils & BONE/SKIN & $L \mid$ Root & D & $\mathrm{O}$ \\
\hline MS $84 / 04$ & $\begin{array}{l}\text { Cycas circinalis } L \\
{[34,35,38,42,43,46,47,51,73]}\end{array}$ & Cycadaceae & Malcoku/Malok & Sores & SKIN & Seed & $\mathrm{R}$ & T \\
\hline BK 002/06 & Cycas rumphii Miq [64] & Cycadaceae & Malehohong & Sores & SKIN & Seed & R & $\mathrm{T}$ \\
\hline GW 90/04 & $\begin{array}{l}\text { Cycas sp. } \\
{[34,38,39,42,43,46,47,51,63,72,73]}\end{array}$ & Cycadaceae & Rarier & Ulcers & SKIN & Seed & $\mathrm{R}$ & T \\
\hline DK 06/05 & Cymbopogon citratus (DC) Stapf $[42,56,66]$ & Gramineae & Suimin & Fever & FEV & Whole & V & $\mathrm{T}$ \\
\hline GW 53/04 & Cymbopogon citratus (DC) Stapf $[42,56,66]$ & Gramineae & Yamawi & Malaria & MAL & $\mathrm{L}$ & V & । \\
\hline MS 70/04 & Davallia sp. $[8,34]$ & Davalliaceae & Klakol & Headache, fever & $\mathrm{HEAD/FEV}$ & Sap & C & $\mathrm{O}$ \\
\hline DK 35/05 & $\begin{array}{l}\text { Dendrocnide cordata (Warb. ex H.J.P. Winkl.) } \\
\text { Chew [51] }\end{array}$ & Urticaceae & Chumbia & Body aches & PAIN & L & $\mathrm{R}$ & T \\
\hline MS 33/04 & Dendrocnide latifolia (Gaudich.) Chew [64] & Urticaceae & Shalat (green) & General body pain & PAIN & L & $\mathrm{R}$ & T \\
\hline GW 101/04 & $\begin{array}{l}\text { Desmodium sp. } \\
{[7,9,33-35,37,43,48,60,66,68,70,72,75,76]}\end{array}$ & Fabaceae & Ninji & Contraceptive & REP & Root & $\mathrm{S}$ & $\mathrm{O}$ \\
\hline MS 81/04 & Dillenia sp. $[39,50,58,60,65,77]$ & Dilleniaceae & Kol & Fever, headache, cough & FEV/HEAD/RESP & $\mathrm{L}$ & S & $\mathrm{O}$ \\
\hline DK 59/05 & Dioscorea bulbifera $L[33,42]$ & Dioscoreaceae & Remsik & Contraceptive & REP & Seed & $\mathrm{s}$ & $\mathrm{O}$ \\
\hline
\end{tabular}


Table 1 Plants reported as medicinally used in 4 study areas in East Sepik Province (Continued)

\begin{tabular}{|c|c|c|c|c|c|c|c|c|}
\hline GW 63/04 & $\begin{array}{l}\text { Dioscorea sp. } \\
{[7,8,33,35,38,42,43,46,54,59,72,73]}\end{array}$ & Dioscoreaceae & Harehare & Headache, migraine & HEAD & $\mathrm{L}$ & $\mathrm{HR}$ & $\mathrm{T}$ \\
\hline BK 013/06 & Donax canniformis (G. Forst.) K. Schum [8] & Marantaceae & Gani & Ear ache & PAIN & $y L$ & R & $\mathrm{T}$ \\
\hline DK 23/05 & Donax canniformis (G. Forst.) K. Schum [8] & Marantaceae & Guarimb & Ear infection & INF & $\mathrm{L}$ & $\mathrm{R}$ & $\mathrm{T}$ \\
\hline GW 78/04 & Dracaena angustifolia (Medik.) Roxb $[42,58]$ & Asparagaceae & Hembesaihe & $\begin{array}{l}\text { Fever, headache, stomach } \\
\text { complaints }\end{array}$ & FEV/HEAD/GAST & Root & S & $\mathrm{O}$ \\
\hline GW 25/04 & Dysoxylum sp. $[33,34,39,41,51,59,63]$ & Meliaceae & Sengiwama & Sores, ulcers & SKIN & B & $\mathrm{R}$ & $\mathrm{T}$ \\
\hline GW 68/04 & Dysoxylum sp. $[33,34,39,41,51,59,63]$ & Meliaceae & Huambuka & Malaria, cough & MAL/RESP & $\mathrm{L}$ & $\mathrm{D}$ & $\mathrm{O}$ \\
\hline GW 100/04 & Elaeocarpus sphaericus Schum [39, 53] & Elaeocarpaceae & Nangila & $\begin{array}{l}\text { Malaria, cough, pneumonia, } \\
\text { shortness of breath }\end{array}$ & MAL/RESP & B & D & $\mathrm{O}$ \\
\hline MS 25/04 & Elaeocarpus sphaericus Schum $[39,53]$ & Elaeocarpaceae & Kaiboun & Asthma & RESP & B & S & $\mathrm{O}$ \\
\hline BK 043/06 & Elatostema sp $[8,33-35,39-41,43,53,65,66,69]$ & Urticaceae & Kaskas-bhirs & Scabies & SKIN & Whole & $\mathrm{D}$ & $\mathrm{T}$ \\
\hline MS 59/04 & Elatostema sp $[8,33-35,39-41,43,53,65,66,69]$ & Urticaceae & Moin kukuri & Fever, headache, joint pain, fertility & $\begin{array}{l}\text { FEV/HEAD/PAIN/ } \\
\text { REP }\end{array}$ & Whole & M & $\mathrm{O}$ \\
\hline GW 28/04 & $\begin{array}{l}\text { Endospermum formicarium Becc } \\
{[7,34,39,43,62,67]}\end{array}$ & Euphorbiaceae & Bundua & Fever, asthma & FEV/RESP & B & S & $\mathrm{O}$ \\
\hline DK 40/05 & $\begin{array}{l}\text { Endospermum labios Schodde } \\
{[7,34,39,43,62,67]}\end{array}$ & Euphorbiaceae & Paruang & Scabies & SKIN & $\begin{array}{l}\text { Seed \& } \\
\text { Flower }\end{array}$ & C & $\mathrm{O}$ \\
\hline MS $89 / 04$ & Endospermum medullosum L.S.Sm. & Euphorbiaceae & Kakar & Fever, body pain, unconscious & FEV/PAIN/PSYCH & L & B & । \\
\hline GW 47/04 & $\begin{array}{l}\text { Epipremnum pinnatum (L.) Engl } \\
{[7,8,34,39,42,67]}\end{array}$ & Araceae & Kumbui-bhi & Fever & FEV & B & $\mathrm{S}$ & $\mathrm{O}$ \\
\hline BK 009/06 & Epipremnum sp. $[7,8,34,39,42,43,66,67]$ & Araceae & Kunga & $\begin{array}{l}\text { Dysentery (excreting of blood), } \\
\text { vomiting of blood }\end{array}$ & GAST & Root & $\mathrm{M} \mid \mathrm{C}$ & $\mathrm{O}$ \\
\hline MS $12 / 04$ & Epipremnum sp. $[7,8,34,39,42,43,66,67]$ & Araceae & Klakial & $\begin{array}{l}\text { Headache, swollen bodies, fever, } \\
\text { cold }\end{array}$ & $\begin{array}{l}\text { HEAD/SWELL/ } \\
\text { FEV/RESP }\end{array}$ & Sap & $\mathrm{S}$ & $\mathrm{O}$ \\
\hline GW 18/04 & Erythrina merrilliana Krukoff & Fabaceae & Kwai & Diarrhoea, shortness of breath,cough & GAST/RESP & $L \& B$ & $\mathrm{D}$ & $\mathrm{O}$ \\
\hline MS 42/04 & Erythrina merrilliana Krukoff & Fabaceae & Pear & Contraceptive & REP & B & B & $\mathrm{O}$ \\
\hline MS 52/04 & $\begin{array}{l}\text { Euodia hortensis J.R. Forst. \& G. Forst. } \\
{[8,34,42,43,53,56,57,63]}\end{array}$ & Rutaceae & Ghin & Unconsciousness & PSYCH & L & V & । \\
\hline MS 66/04 & Euodia sp. $[8,33,34,40,42,43,53,56,57,62,63]$ & Rutaceae & Muth & Fertility,emetic & REP/GAST & B & S & $\mathrm{O}$ \\
\hline BK 025/06 & Euphorbia heterophylla L [51] & Euphorbiaceae & Wilai & For treating diarrhoea & GAST & Sap & S & $\mathrm{O}$ \\
\hline BK 023/06 & Euphorbia hirta L $[9,34,39,46,50,51,56,57,67]$ & Euphorbiaceae & Unknown & Sore & SKIN & $L$ & $\mathrm{~S}$ & $\mathrm{~T}$ \\
\hline GW 17/04 & Euphorbia hirta L $[9,34,39,46,50,51,56,57,67]$ & Euphorbiaceae & Seplein Nai & $\begin{array}{l}\text { Shortness of breath, asthma, } \\
\text { pneumonia }\end{array}$ & RESP & Whole & D & $\mathrm{O}$ \\
\hline DK 03/05 & $\begin{array}{l}\text { Euphorbia plumerioides Teijsm. ex Hassk. } \\
{[33,34,36,43,51,53,60,69]}\end{array}$ & Euphorbiaceae & Miambi/Pombi & Poisoning & POIS & Sap & S & $\mathrm{O}$ \\
\hline MS 47/04 & $\begin{array}{l}\text { Euphorbia sp. } \\
{[9,34-36,41-44,50,53,54,57-59,67,74,78]}\end{array}$ & Euphorbiaceae & Sungwia & Emetic & GAST & Sap & S & $\mathrm{O}$ \\
\hline
\end{tabular}


Table 1 Plants reported as medicinally used in 4 study areas in East Sepik Province (Continued)

\begin{tabular}{|c|c|c|c|c|c|c|c|c|}
\hline GW 44/04 & $\begin{array}{l}\text { Euphorbia sp. } \\
{[9,34-36,41-44,50,53,54,57-59,67,74,78]}\end{array}$ & Euphorbiaceae & Wale & Emetic & GAST & Sap & $S$ & $\mathrm{O}$ \\
\hline GW 80/04 & $\begin{array}{l}\text { Euphorbia sp. } \\
{[9,34-36,41-44,50,53,54,57-59,67,74,78]}\end{array}$ & Euphorbiaceae & Tuth & Emetic & GAST & Sap & S & $\mathrm{O}$ \\
\hline MS $79 / 04$ & Euphorbia tithymaloides (L.) $[51,56]$ & Euphorbiaceae & Mual nias & Epigastric pain & GAST & Sap & S & $\mathrm{O}$ \\
\hline BK 046/06 & Ficus adenosperma Miq $[8,33-35,53]$ & Moraceae & Belloki & Cut & WOUND & $y L$ & S & $\mathrm{T}$ \\
\hline DK 41/05 & $\begin{array}{l}\text { Ficus pungens Reinw. ex Blume } \\
{[9,33,34,42,43,53,59,60,63]}\end{array}$ & Moraceae & Kuar & Shortness of breath & RESP & Succus & $S$ & $\mathrm{O}$ \\
\hline MS 40/04 & $\begin{array}{l}\text { Ficus septica Burm.f. } \\
{[34,37-40,42,43,48,49,51,57-59,61,62 \text {, }} \\
66,67,78]\end{array}$ & Moraceae & Poipuk & Diarrhoea & GAST & $\begin{array}{l}\text { Sap \& } \\
\text { yShoot }\end{array}$ & S & $\mathrm{O}$ \\
\hline DK 58/05 & $\begin{array}{l}\text { Ficus sp. } \\
{[7-9,33-40,42-45,47-49,51-59,61-63,66-69} \\
74,75,77,78]\end{array}$ & Moraceae & Tuohepolehe & Malnutrition & NUT & Sap & C & $\mathrm{O}$ \\
\hline GW 58/04 & $\begin{array}{l}\text { Ficus sp. }[7-9,33-40,42-45,47-49,51-59, \\
61-63,66-69,74,75,77,78]\end{array}$ & Moraceae & Manjemieri & Nutrient supplement for babies & NUT & Sap & S & $\mathrm{O}$ \\
\hline GW 74/04 & $\begin{array}{l}\text { Ficus sp. }[7-9,33-40,42-45,47-49,51-59, \\
61-63,66-69,74,75,77,78]\end{array}$ & Moraceae & $\begin{array}{l}\text { Wavihasa/ } \\
\text { Horikieng }\end{array}$ & Broken bones & BONE & Root & M & T \\
\hline GW 89/04 & $\begin{array}{l}\text { Ficus sp. }[7-9,33-40,42-45,47-49,51-59, \\
61-63,66-69,74,75,77,78]\end{array}$ & Moraceae & Chiplapul & Abortion & REP & B & R & T \\
\hline MS $17 / 04$ & $\begin{array}{l}\text { Ficus sp. }[7-9,33-40,42-45,47-49,51-59, \\
61-63,66-69,74,75,77,78]\end{array}$ & Moraceae & Bukabok & Fracture & BONE & B & $\mathrm{R}$ & T \\
\hline MS 31/04 & $\begin{array}{l}\text { Ficus sp. }[7-9,33-40,42-45,47-49,51-59, \\
61-63,66-69,74,75,77,78]\end{array}$ & Moraceae & Moul koni & Ulcer & SKIN & Sap & S & T \\
\hline MS $88 / 04$ & $\begin{array}{l}\text { Ficus sp. }[7-9,33-40,42-45,47-49,51-59, \\
61-63,66-69,74,75,77,78]\end{array}$ & Moraceae & Aiyau & Toothache & DENT & yRoot & M & $\mathrm{O}$ \\
\hline MS 75/04 & Ficus wassa Roxb $[33,34,39,40,42,47,68,75]$ & Moraceae & Kikquai & Contraceptive & REP & Root & M & $\mathrm{O}$ \\
\hline BK 060/06 & Gnetum gnemon $L[8,34]$ & Gnetaceae & Yit & Removal of wood or stick in skin & WOUND & $y L$ & S & T \\
\hline DK 14/05 & Gnetum gnemon L $[8,34]$ & Gnetaceae & Mogsa & $\begin{array}{l}\text { Removal of nails/ splints lodged in } \\
\text { the body }\end{array}$ & WOUND & Sap & S & $\mathrm{T}$ \\
\hline MS 18/04 & Gnetum gnemon L $[8,34]$ & Gnetaceae & Popoyiri & Eye disease & OCC & Sap & S & T \\
\hline GW 45/04 & Gnetum gnemonoides Brongn. & Gnetaceae & Biek & Fever, headache (malaria) & FEV/MAL & B & $\mathrm{D}$ & $\mathrm{O}$ \\
\hline MS $14 / 04$ & $\begin{array}{l}\text { Graptophyllum sp. } \\
{[7-9,33,35,36,39,41,66,67]}\end{array}$ & Acanthaceae & Inta'niat & Fever, headache, joint pain, cold & $\begin{array}{l}\text { FEV/HEAD/ } \\
\text { PAIN/RESP }\end{array}$ & $\mathrm{L}$ & $\mathrm{D}$ & $O \& \mid \& T$ \\
\hline GW 11/04 & $\begin{array}{l}\text { Gymnostoma papuanum (S. Moore) L.A.S. Johnson } \\
{[33,35,43]}\end{array}$ & Casuarinaceae & Mania & Shortness of breath, asthma & RESP & B & D & $\mathrm{O}$ \\
\hline GW 70/04 & $\begin{array}{l}\text { Hemigraphis reptans (G. Forst.) T. Anderson ex } \\
\text { Hemsl. }\end{array}$ & Acanthaceae & Mijika & Centipede bite & BITE & Whole & $H R$ & $\mathrm{~T}$ \\
\hline BK 018/06 & Hibiscus rosa-sinensis $L[37,56,59]$ & Malvaceae & Mawe & Sore eye & OCC & Flower & $\mathrm{R}$ & $\mathrm{T}$ \\
\hline
\end{tabular}


Table 1 Plants reported as medicinally used in 4 study areas in East Sepik Province (Continued)

\begin{tabular}{|c|c|c|c|c|c|c|c|c|}
\hline DK 13/05 & Hibiscus rosa-sinensis $L[37,56,59]$ & Malvaceae & Kupawaruk & Menstrual cramps & REP & L & S & $\mathrm{O}$ \\
\hline MS 05/04 & Homalanthus sp. $[7,8,33-36,42,50,51,58]$ & Euphorbiaceae & War moap & Scabies & SKIN & Stem & $\mathrm{D}$ & T \\
\hline DK 42/05 & Homalium foetidum (Roxb.) Benth [8] & Salicaceae & Mes & Knee ache & PAIN & B & MAG & P_to_Plant \\
\hline GW 83/04 & Hydriastele costata F.M. Bailey & Arecaceae & Yawah & Shortness of breath & RESP & Stem & S & $\mathrm{O}$ \\
\hline DK 33/05 & Intsia bijuga (Colebr.) Kuntze & Fabaceae & Wun & Boil & SKIN & Stem & C & $\mathrm{T}$ \\
\hline GW 08/04 & Intsia bijuga (Colebr.) Kuntze & Fabaceae & Hwapo & Fractured bones & BONE & B & $\mathrm{HR}$ & T \\
\hline MS 46/04 & Intsia bijuga (Colebr.) Kuntze & Fabaceae & Tou'r & Severe back pain & PAIN & B & D & $O \& T$ \\
\hline GW 52/04 & Ipomea sp. $[35,36,39,43,49,61]$ & Convolvulaceae & Firac & Distended stomach, pigbel & GAST & L & C & O \\
\hline BK 020/06 & Ipomoea pes-caprae (L.) R. Br [59] & Convolvulaceae & Waimabhu & Running nose, cough, asthma & RESP & Stem & S & $?$ \\
\hline MS 26/04 & Ipomoea pes-caprae (L.) R. Br [59] & Convolvulaceae & Kairo & $\begin{array}{l}\text { Fever, headache, joint pain, swelling } \\
\text { of the body }\end{array}$ & $\begin{array}{l}\text { FEV/HEAD/PAIN/ } \\
\text { SWELL }\end{array}$ & L & S & $\mathrm{O}$ \\
\hline BK 021/06 & Kalanchoe pinnata (Lam.) Pers $[34,39,46,51,67]$ & Crassulaceae & Kulukir & $\begin{array}{l}\text { knee pain/ache, back ache/pain, } \\
\text { swollen legs, boils }\end{array}$ & $\begin{array}{l}\text { PAIN/ SWELL } \\
\text { /SKIN }\end{array}$ & L & $\mathrm{H}$ & T \\
\hline DK 04/05 & Kalanchoe pinnata (Lam.) Pers $[34,39,46,51,67]$ & Crassulaceae & Asamambia & Insect bite & BITE & L & $\mathrm{H}$ & $\mathrm{T}$ \\
\hline BK 015/06 & $\begin{array}{l}\text { Laportea decumana Wedd. } \\
{[9,32,34-37,39,42-45,49,51,53,59,63,65,66,} \\
69,71,72,74-77]\end{array}$ & Urticaceae & Salat & $\begin{array}{l}\text { Muscle ache, knee pain, ankle } \\
\text { sprain }\end{array}$ & PAIN & L & $\mathrm{R}$ & $\mathrm{T}$ \\
\hline DK 32/05 & Laportea interrupta (L.) Chew $[34,39,51,67,73]$ & Urticaceae & Shalat (red) & Fresh cuts, wounds & WOUND & $y L$ & $\mathrm{H}$ & $\mathrm{T}$ \\
\hline BK 027/06 & Leucosyke capitellata Wedd $[8,9]$ & Urticaceae & Elan & Sores and cuts & SKIN/WOUND & B & $\mathrm{R}$ & T \\
\hline GW 22/04 & Litsea sp. $[8,9,33,43,69]$ & Lauraceae & Erikombi & Cough, malaria & RESP/MAL & L & $\mathrm{D}$ & $\mathrm{O}$ \\
\hline GW 23/04 & Litsea sp. $[8,9,33,43,69]$ & Lauraceae & Neimie & Malaria, fevers, coughs & MAL/FEV/RESP & $\mathrm{L}$ or $\mathrm{B}$ & $\mathrm{D}$ & $\mathrm{O}$ \\
\hline GW 06/04 & Macaranga clavata Warb. & Euphorbiaceae & Lambie & Skin infections, scabies & SKIN & B & $\mathrm{S}$ & T \\
\hline MS $11 / 04$ & Macaranga darbyshirei Airy Shaw & Euphorbiaceae & Walmieng & Anti-venom & POIS & B & M & $\mathrm{O}$ \\
\hline GW 46/04 & Maclura cochinchinensis (Lour.) Corner [39] & Moraceae & Lomowi & Cough, stomach complaints & RESP/GAST & Stem & S & $\mathrm{O}$ \\
\hline GW 93/04 & Mangifera indica $L[8,34,51,56]$ & Anacardiaceae & $\begin{array}{l}\text { Huarambie/ } \\
\text { Wamahang }\end{array}$ & Snake bite & BITE & B & $\mathrm{D} \mid \mathrm{H}$ & $O \& T$ \\
\hline DK 51/05 & Manihot esculenta Crantz $[34,35,51]$ & Euphorbiaceae & Gumbyow & Fresh cut,wounds & WOUND & Root & R & T \\
\hline MS 16/04 & Marattia sp. $[7,33,34,36,43,54,69]$ & Marattiaceae & Rireo & $\begin{array}{l}\text { Fever, headache, swollen bodies } \\
\text { etc. }\end{array}$ & $\begin{array}{l}\text { FEV/HEAD/ } \\
\text { SWELL/OTHER }\end{array}$ & yShoot & S & $\mathrm{O}$ \\
\hline BK 011/06 & $\begin{array}{l}\text { Melanolepis multiglandulosa (Reinw. ex Blume) } \\
\text { Rchb. \& Zoll [34] }\end{array}$ & Euphorbiaceae & Wamakhir & Snake bites & BITE & B & M & $\mathrm{O}$ \\
\hline DK 36/05 & $\begin{array}{l}\text { Melanolepis multiglandulosa (Reinw. ex Blume) } \\
\text { Rchb. \& Zoll [34] }\end{array}$ & Euphorbiaceae & Waru & Snake bite & BITE & B & M & $\mathrm{O}$ \\
\hline GW 02/04 & $\begin{array}{l}\text { Melanolepis multiglandulosa (Reinw. ex Blume) } \\
\text { Rchb. \& Zoll [34] }\end{array}$ & Euphorbiaceae & Warimaing & $\begin{array}{l}\text { Snake and centipede bites, } \\
\text { antivenom }\end{array}$ & BITE/POIS & B & M & $\mathrm{O}$ \\
\hline MS 36/04 & Melastoma sp. [7-9, 33, 34, 43, 47, 54] & Melastomataceae & Mutamuth & Blocked nose, flu, cough & RESP & L & V & । \\
\hline DK 05/05 & Melicope triphylla (Lam.) Merr [34, 40, 42, 43, 62] & Rutaceae & Kupun & Abortion & REP & $\mathrm{L}$ & D & 0 \\
\hline
\end{tabular}


Table 1 Plants reported as medicinally used in 4 study areas in East Sepik Province (Continued)

\begin{tabular}{|c|c|c|c|c|c|c|c|c|}
\hline BK 001/06 & Merremia peltata (L.) Merr $[8,34,42,43,56,59,67]$ & Convolvulaceae & Aukut & Boil, sore or ulcer, fresh cut & SKIN/WOUND & Sap $\mid L$ & $\mathrm{~S} \mid \mathrm{H}$ & $\mathrm{T}$ \\
\hline DK 28/05 & Merremia peltata (L.) Merr $[8,34,42,43,56,59,67]$ & Convolvulaceae & Bangpuk & Fresh cuts, /wounds & WOUND & Sap & $\mathrm{s}$ & $\mathrm{T}$ \\
\hline GW 62/04 & Merremia peltata (L.) Merr $[8,34,42,43,56,59,67]$ & Convolvulaceae & Nangumareng & Determine male sex of baby & REP & $\mathrm{L}$ & $\mathrm{D}$ & $\mathrm{O}$ \\
\hline GW 43/04 & Merremia sp. $[8,34,42,43,56,59,67]$ & Convolvulaceae & Wararamang & Fever, malaria & FEV/MAL & Stem & S & $\mathrm{O}$ \\
\hline DK 30/05 & Metroxylon sagu Rottb [42] & Arecaceae & Nouk & Burns & BURN & Stem & $\mathrm{R}$ & $\mathrm{T}$ \\
\hline GW 96/04 & Mikania sp. & Asteraceae & Lihasuanga & Skin infections, scabies, sores & SKIN & Sap & S & $\mathrm{T}$ \\
\hline BK 059/06 & Mimosa pudica L $[8,34]$ & Fabaceae & Bambu kiya & Induce sleep & PSYCH & Whole & $\mathrm{D}$ & $\mathrm{T}$ \\
\hline DK 52/05 & Mimosa pudica L $[8,34]$ & Fabaceae & Haihiksa & Infant colic & CHILD & Whole & $\mathrm{D}$ & $\mathrm{T}$ \\
\hline MS 77/04 & Mimosa pudica L $[8,34]$ & Fabaceae & Miatmiat & Induced sleep & PSYCH & Whole & $\mathrm{D}$ & $\mathrm{T}$ \\
\hline GW 20/04 & Mitracarpus sp. & Rubiaceae & Waramang & Eye infections, color defects & OCC & Whole & B & 1 \\
\hline BK 038/06 & $\begin{array}{l}\text { Morinda citrifolia L }[7,8,34,37,38,42,43,48,49 \\
56-59,61,68,70,73,78]\end{array}$ & Rubiaceae & Simbiya & Knee ache, cough & PAIN/RESP & $y L \mid$ Fruit & $\underset{H}{D} \mid R$ or & $O \& T$ \\
\hline MS 71/04 & $\begin{array}{l}\text { Morinda citrifolia L }[7,8,34,37,38,42,43,48,49 \\
56-59,61,68,70,73,78]\end{array}$ & Rubiaceae & Knuel & $\begin{array}{l}\text { General body pain, boils, } \\
\text { inflammation }\end{array}$ & $\begin{array}{l}\text { PAIN/SKIN/ } \\
\text { SWELL }\end{array}$ & $\mathrm{L}$ & $\mathrm{R}$ & $\mathrm{T}$ \\
\hline GW 64/04 & Mucuna novo-guineensis Scheff. [8] & Fabaceae & Kilemiesik & Shortness of breath & RESP & Root & S & $\mathrm{O}$ \\
\hline MS 37/04 & Mucuna sp. $[8,9,34,43,47,50,52,56,63]$ & Fabaceae & Ombo & Anemia & BLOOD & Sap & S & $\mathrm{O}$ \\
\hline GW 51/04 & Mucuna sp. $[8,9,34,43,47,50,52,56,63]$ & Fabaceae & Wamayihara & Tooth ache, loose tooth & DENT & Stem & M & $\mathrm{O}$ \\
\hline GW 66/04 & Mucuna sp. $[8,9,34,43,47,50,52,56,63]$ & Fabaceae & Ponambile & Anemia & BLOOD & B & $\mathrm{s}$ & $\mathrm{O}$ \\
\hline GW 84/04 & Mucuna sp. $[8,9,34,43,47,50,52,56,63]$ & Fabaceae & Manvil & Arthritis joint pain, back ache & PAIN & B & $\mathrm{S}$ & $\mathrm{T}$ \\
\hline BK 003/06 & Murraya paniculata (L.) Jack $[8,73]$ & Rutaceae & Sika & Ccough & RESP & $\mathrm{L}$ & $\mathrm{D}$ & $\mathrm{O}$ \\
\hline DK 24/05 & Musa acuminata Colla $[34,37,47,57]$ & Musaceae & Yup & Sore lip & PAIN & Fruit & C & $\mathrm{T}$ \\
\hline MS 44/04 & $\begin{array}{l}\text { Musa sp. }[8,9,32-34,37,42,43,47,52,54,56 \\
57,59,61,63,67,71,72]\end{array}$ & Musaceae & Wur karasau & Wound & WOUND & Sap & S & $\mathrm{T}$ \\
\hline BK 055/06 & Nauclea orientalis $(\mathrm{L}.) \mathrm{L}[34,55,63]$ & Rubiaceae & Runggool & Asthma,shortness of breath & RESP & B & S & $\mathrm{O}$ \\
\hline DK 44/05 & Nauclea orientalis $(\mathrm{L}.) \mathrm{L}[34,55,63]$ & Rubiaceae & Kuva & Snake bite & BITE & B & $\mathrm{S}$ & $\mathrm{O}$ \\
\hline GW 10/04 & Neonauclea purpurea (Roxb.) Merr [39] & Rubiaceae & Kripa & $\begin{array}{l}\text { Fever, headache (malaria), } \\
\text { pneumonia, asthma }\end{array}$ & FEV/MAL/RESP & B & B & $1 \& O$ \\
\hline BK 061/06 & Neonauclea sp. & Rubiaceae & Gipma & Poisonous snake bite & BITE & B & M & $\mathrm{O}$ \\
\hline DK 48/05 & Nephrolepis hirsutula (G. Forst.) C. Presl [8] & Lomariopsidaceae & Tamanguia & Uncontrollable urine & URINE & $\mathrm{L}$ & C & $\mathrm{O}$ \\
\hline GW 36/04 & Nephrolepis sp. [7, 8, 33, 34, 43] & Lomariopsidaceae & Walendau & Headache, fever (malaria) & HEAD/MAL & $\begin{array}{l}\text { Shoot } \\
\text { \& Root }\end{array}$ & S & $\mathrm{O}$ \\
\hline MS 48/04 & $\begin{array}{l}\text { Nicotiana sp. } \\
{[33-36,42,43,47,52,59,63,66,71,72,76,77]}\end{array}$ & Solanaceae & Kennings & Anticoagulant & BLOOD & $y L$ & $\mathrm{H}$ & $\mathrm{T}$ \\
\hline BK 024/06 & $\begin{array}{l}\text { Nicotiana tabacum (L.) } \\
{[33,35,36,42,43,52,59,63,76,77]}\end{array}$ & Solanaceae & Saukien & Sores & SKIN & L & S & $\mathrm{T}$ \\
\hline BK 036/06 & Not Identified & Not Identified & Ukapuk & Scabies, malaria & SKIN/MAL & Sap & $\mathrm{S}$ & $\mathrm{T} \mid \mathrm{O}$ \\
\hline
\end{tabular}


Table 1 Plants reported as medicinally used in 4 study areas in East Sepik Province (Continued)

\begin{tabular}{|c|c|c|c|c|c|c|c|c|}
\hline DK 47/05 & Not identified & Not identified & Kupnenj & Shortness of breath & RESP & Succus & S & $\mathrm{O}$ \\
\hline DK 60/05 & Not identified & Fabaceae & Wulamian & Malnutrition & NUT & Whole & $\mathrm{H}$ & $\mathrm{O}$ \\
\hline MS 80/04 & Not identified & Orchidaceae & Kraufung & Skin disease (grille) & SKIN & L & $\mathrm{H}$ & T \\
\hline DK 57/05 & Not identified & Not identified & Sarimbiya & Cough & RESP & $\mathrm{L}$ & - & $\mathrm{O}$ \\
\hline MS 22/04 & Not identified & Not identified & Asakurkunja & Scabies & SKIN & $\begin{array}{l}\text { Stem \& } \\
\text { Root }\end{array}$ & D & T \\
\hline MS 08/04 & $\begin{array}{l}\text { Ocimum basilicum } L \\
{[32,34,38,39,42,43,46,48,65,67,73]}\end{array}$ & Labiate & Ruk & $\begin{array}{l}\text { General body weakness, fever, } \\
\text { headache, etc. }\end{array}$ & FEV/MAL/HEAD & Whole & B & 1 \\
\hline BK 004/06 & Octomeles sumatrana Miq [43] & Datiscaceae & Wani & $\begin{array}{l}\text { Asthma, back ache, } \\
\text { malnourished/pigbel }\end{array}$ & $\begin{array}{l}\text { RESP/PAIN/NUT/ } \\
\text { GAST }\end{array}$ & B | B | Sap & 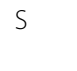 & $0|O| O$ \\
\hline GW 48/04 & Octomeles sumatrana Miq [43] & Datiscaceae & Waine & Fever & FEV & B & S & $\mathrm{O}$ \\
\hline MS 30/04 & Pandanus dubius Spreng. & Pandanaceae & Viak & Asthma & RESP & yShoot & S & $\mathrm{O}$ \\
\hline GW 98/04 & Pangium edule Reinw $[34,42,43,50,51,53]$ & Achariaceae & Imahek & Enlarged spleen & ORG & Fruit & R & $\mathrm{O}$ \\
\hline MS 35/04 & Pangium edule Reinw $[34,42,43,50,51,53]$ & Achariaceae & Sis & Lice killer & INSECTICIDE & L & S & T \\
\hline GW 65/04 & Papuechites sp. $[34,43]$ & Apocynaceae & Pari & Enlarged spleen & ORG & Fruit & S & O \\
\hline GW 29/04 & Parsonia sp. $[57,59]$ & Apocynaceae & Tielimbika & Fresh cuts, sores & SKIN/WOUND & $\mathrm{L}$ & $\mathrm{H}$ & $\mathrm{T}$ \\
\hline BK 032/06 & Passiflora foetida L $[8,42,51,56]$ & Passifloraceae & Bombo & Asthma, white spot & RESP/SKIN & $\begin{array}{l}\text { Flower \& L } \\
\text { | Seed }\end{array}$ & $D \mid R$ & $O \& T$ \\
\hline DK 46/05 & Passiflora foetida $L[8,42,51,56]$ & Passifloraceae & Apsarapuk & Whitespots & SKIN & L & $\mathrm{R}$ & T \\
\hline GW 19/04 & Passiflora foetida $L[8,42,51,56]$ & Passifloraceae & Apduanpuk & Strong cough & RESP & Shoot \& L & S & $\mathrm{O}$ \\
\hline MS 09/04 & Passiflora foetida $L[8,42,51,56]$ & Passifloraceae & Maparou & Skin disease & SKIN & & $\mathrm{R}$ & T \\
\hline DK 55/05 & Passiflora sp. $[8,42,51,56]$ & Passifloraceae & War yasokk & Scabies & SKIN & Sap & $\mathrm{H}$ & $\mathrm{T}$ \\
\hline BK 017/06 & Peperomia pellucida (L.) Kunth [7] & Piperaceae & Koikoiwara & Pimple & SKIN & L & $\mathrm{R}$ & $\mathrm{T}$ \\
\hline GW 81/04 & Peperomia pellucida (L.) Kunth [7] & Piperaceae & Lerek & Fever, headache, (malaria) & FEV/MAL & Whole & D & $\mathrm{O}$ \\
\hline MS 58/04 & Peperomia pellucida (L.) Kunth [7] & Piperaceae & Kinkanak & Antidepressant & PSYCH & L & D & $\mathrm{T}$ \\
\hline BK 014/06 & Phrynium sp. [45] & Marantaceae & Ripa kwalingu & Scabies & SKIN & Succus & S & $\mathrm{T}$ \\
\hline MS 55/04 & $\begin{array}{l}\text { Phyllanthus amarus Schumach. \& Thonn } \\
{[34,43,49,52,63]}\end{array}$ & Phyllanthaceae & Kambaningi & Fever, headache, swollen bodies & $\begin{array}{l}\text { FEV/HEAD/ } \\
\text { SWELL }\end{array}$ & Root & S & $\mathrm{O}$ \\
\hline GW 54/04 & Phyllanthus niruri L $[7,9,34,42,43,46,57,59,73]$ & Phyllanthaceae & Hipanchinchi & Menorrhagia & REP & Whole & D & $\mathrm{O}$ \\
\hline MS 60/04 & Phyllanthus niruri L $[7,9,34,42,43,46,57,59,73]$ & Phyllanthaceae & Shuk miau & Fever & FEV & Whole & $\mathrm{D}$ & $\mathrm{T}$ \\
\hline GW 14/04 & $\begin{array}{l}\text { Phyllanthus sp. } \\
{[7,9,33-35,39,42,43,46,49,52,57,59,63,67,73]}\end{array}$ & Phyllanthaceae & Kai veai & Tooth infections, toothache & DENT & Root & M & T \\
\hline GW 61/04 & Pimelodendron amboinicum Hassk [34, 39] & Euphorbiaceae & Sombik & Enlarged spleen & ORG & Sap & S & $\mathrm{O}$ \\
\hline MS 15/04 & Pimelodendron amboinicum Hassk [34, 39] & Euphorbiaceae & Kunial & Swollen stomach & GAST & B & $D$ & $\mathrm{~T}$ \\
\hline BK 062/06 & Piper betle $\mathrm{L}[37-39,42,61,67,68]$ & Piperaceae & Kwashe gungga & Sores,boils & SKIN & L & $\mathrm{H}$ & $\mathrm{T}$ \\
\hline
\end{tabular}


Table 1 Plants reported as medicinally used in 4 study areas in East Sepik Province (Continued)

\begin{tabular}{|c|c|c|c|c|c|c|c|c|}
\hline DK 22/05 & Piper betle L $[37-39,42,61,67,68]$ & Piperaceae & Kosh & Abdominal ache & GAST & Seed & MS & $\mathrm{T}$ \\
\hline GW 49/04 & Piper betle L $[37-39,42,61,67,68]$ & Piperaceae & Guspui & Tuberculosis, centipede bite & BITE/INF & $L \mid$ Fruit & $\mathrm{H}$ & $\mathrm{O} \mid \mathrm{T}$ \\
\hline DK 27/05 & Piper mestonii F.M. Bailey. & Piperaceae & Hrunga & Fresh cuts, wounds & WOUND & $\mathrm{L}$ & S & $\mathrm{T}$ \\
\hline GW 97/04 & $\begin{array}{l}\text { Piper sp. }[8,9,33,34,37-39,42,43,53,55,57,58, \\
60,61,65,67,68,70,74]\end{array}$ & Piperaceae & Walehru & Memory enhancing, clear thinking & PSYCH & Root & M & $\mathrm{O}$ \\
\hline MS 56/04 & $\begin{array}{l}\text { Piper sp. }[8,9,33,34,37-39,42,43,53,55,57,58 \text {, } \\
60,61,65,67,68,70,74]\end{array}$ & Piperaceae & Kunek & Anesthetic & PAIN & Root & S & $\mathrm{O}$ \\
\hline DK 31/05 & Piscidia grandifolia (Donn. Sm.) I.M. Johnst. [8] & Fabaceae & Yinapuk & Strong headache & HEAD & Stem & $\mathrm{R}$ & $\mathrm{T}$ \\
\hline GW 32/04 & Pisonia longirostris Teijsm. \& Binn [56] & Nyctaginaceae & Kumie/Weworo & Tropical ulcers, peptic ulcers & SKIN/GAST & B & S & $\mathrm{T} \mid \mathrm{O}$ \\
\hline DK 45/05 & Planchonia papuana R. Knuth & Lecythidaceae & Ningia & Scabies & SKIN & B & C & $\mathrm{O}$ \\
\hline MS 57/04 & Plectranthus amboinicus (Lour.) Spreng $[60,74]$ & Labiatae & Wasirika & Skin disease (grille) & SKIN & L & S & $\mathrm{T}$ \\
\hline GW 13/04 & Plectranthus hereroensis Engl. & Labiatae & Sumoun & Stomach ulcers, placenta sores & GAST/REP & $\mathrm{L}$ & $\mathrm{D}$ & $\mathrm{O}$ \\
\hline GW 15/04 & Plectranthus hereroensis Engl. & Labiatae & Krau sumin & Scabies, itchy skin & SKIN & L & S & $\mathrm{T}$ \\
\hline BK 031/06 & Plectranthus parviflorus Willd. & Labiatae & Humbiang & Sores & SKIN & $\mathrm{L}$ & S & $\mathrm{T}$ \\
\hline MS 49/04 & $\begin{array}{l}\text { Plectranthus scutellarioides (L.) R.Br }[8,9,33,42 \text {, } \\
43,56,58,74]\end{array}$ & Labiatae & Humbiang & Ulcer, fresh cut & SKIN/WOUND & $\mathrm{L}$ & $S$ & $\mathrm{~T}$ \\
\hline MS 87/04 & $\begin{array}{l}\text { Plectranthus scutellarioides(L.) R.BR }[8,9,33,42 \text {, } \\
43,56,58,74]\end{array}$ & Labiatae & Trakain & Skin disease (grille) & SKIN & $\mathrm{L}$ & $\mathrm{R}$ & $\mathrm{T}$ \\
\hline GW 30/04 & $\begin{array}{l}\text { Pongamia pinnata (L.) Pierre }[40,42,43,52,56 \text {, } \\
59,63]\end{array}$ & Fabaceae & Lai & Skin infections, scabies & SKIN & Root & S & $\mathrm{T}$ \\
\hline GW 41/04 & Pouteria sp. & Sapotaceae & Pokware & Scabies, grille & SKIN & Sap & $S$ & $\mathrm{~T}$ \\
\hline BK 052/06 & Premna serratifolia L. [39, 42, 61] & Lamiaceae & Kunggwia & Emetic & GAST & Seed & $\mathrm{R}$ & $\mathrm{O}$ \\
\hline GW 42/04 & Premna sp. $[8,34,39,42,43,53,56,63]$ & Lamiaceae & Ningrik & Ear ache & PAIN & B & S & $\mathrm{T}$ \\
\hline MS 06/04 & Premna sp. $[8,34,39,42,43,53,56,63]$ & Lamiaceae & Wurweik & Malaria & MAL & $L \& B$ & $\mathrm{D}$ & $\mathrm{O}$ \\
\hline BK 050/06 & Psidium guajava $\mathrm{L}[39,40,56,61]$ & Myrtaceae & Yambu & Cough, itchy throat & RESP & Fruit & $\mathrm{R}$ & $\mathrm{O}$ \\
\hline DK 17/05 & Psidium guajava $L[39,40,56,61]$ & Myrtaceae & Yambosik & Diarrhoea & GAST & $\mathrm{L}$ & $\mathrm{D}$ & $\mathrm{O}$ \\
\hline DK 49/05 & Psychotria sp. $[7,9,33-35,37,38,43,59]$ & Rubiaceae & Sisikupa & Boil & SKIN & L & S & $\mathrm{T}$ \\
\hline GW 07/04 & Psychotria sp. [7, 9, 33-35, 37, 38, 43, 59] & Rubiaceae & Konumbo & Enlarged spleen & ORG & Sap & $\mathrm{s}$ & $\mathrm{O}$ \\
\hline BK 040/06 & $\begin{array}{l}\text { Pterocarpus indicus Willd. }[7,8,34,37,38,40, \\
42,43,47,48,56,57,59,61,67,68]\end{array}$ & Fabaceae & Markulu & Anemia & BLOOD & Sap & S & $\mathrm{O}$ \\
\hline GW 03/04 & $\begin{array}{l}\text { Pterocarpus indicus Willd. } \\
{[7,8,34,37,38,40,42,43,47,48,56,57,59,61,67,68]}\end{array}$ & Fabaceae & Moroho & Diarrhoea, stomach ache, anemia & GAST/BLOOD & $L \& B$ & $D \mid D$ & $\mathrm{O}$ \\
\hline DK $18 / 05$ & Riedelia corallina (K. Schum.) Valeton & Zingiberaceae & Moukuaikuai & Menstrual cramps & REP & Root & $\mathrm{D}$ & $\mathrm{O}$ \\
\hline MS 63/04 & Scaevola sericea Vahl [39] & Goodeniaceae & Knanas & Cough & RESP & $y L$ & $\mathrm{~S}$ & $\mathrm{O}$ \\
\hline MS 83/04 & Schismatoglottis calyptrata (Roxb.) Zoll. \& Moritzi & Araceae & Maghau & Sore & SKIN & $\mathrm{L}$ & $\mathrm{H}$ & $\mathrm{T}$ \\
\hline GW 55/04 & Semecarpus sp. $[8,50,51]$ & Anacardiaceae & Huaho & Itchy skin (pruritis) & SKIN & B & D & $\mathrm{T}$ \\
\hline
\end{tabular}


Table 1 Plants reported as medicinally used in 4 study areas in East Sepik Province (Continued)

\begin{tabular}{|c|c|c|c|c|c|c|c|c|}
\hline MS 76/04 & $\begin{array}{l}\text { Sida rhombifolia } L[7,8,34,37,40,42,43,50,51 \text {, } \\
54,57,59,60,73]\end{array}$ & Malvaceae & Shasar & Contraceptive & REP & Root & M & $\mathrm{O}$ \\
\hline GW 99/04 & Smilax sp. & Smilacaceae & Kilembole & Generalcleansing & MAINT & $\begin{array}{l}\text { Root \& } \\
\text { Stem }\end{array}$ & S & $\mathrm{O}$ \\
\hline GW 26/04 & Solanum torvum Sw $[8,34,42,48,68]$ & Solanaceae & $\begin{array}{l}\text { Warandangu/ } \\
\text { Waramande }\end{array}$ & Joint pains,arthritis & PAIN & Root & $\mathrm{D}$ & $\mathrm{O}$ \\
\hline GW 33/04 & Spathiphyllum sp. & Araceae & Hwembung & Strong cough, fever & RESP/FEV & Root & s & $\mathrm{O}$ \\
\hline GW 95/04 & Sphaerostephanos sp. [7, 8, 33, 42, 53] & Thelypteridaceae & Ningi & Malaria & MAL & Root & $\mathrm{D}$ & $\mathrm{O}$ \\
\hline MS 24/04 & Sphaerostephanos unitus (L.) Holttum $[33,53]$ & Thelypteridaceae & Kipokip & Sores, ulcers & SKIN & L & S & T \\
\hline DK 50/05 & Spondias dulcis Parkinson [43] & Anacardiaceae & Nungwi & Scabies & SKIN & B & C & $\mathrm{O}$ \\
\hline GW 67/04 & Spondias dulcis Parkinson [43] & Anacardiaceae & Akanang & Sores, scabies & SKIN & Shoot & S & O \\
\hline GW 37/04 & Stephania japonica var. discolor (Blume) Forman [51] & Menispermaceae & Poponga & Malaria & MAL & Root & S & O \\
\hline GW 31/04 & Stephania sp. $[34,37,38,43,47,48,51]$ & Menispermaceae & $\begin{array}{l}\text { Yuamareng/ } \\
\text { Kenduek }\end{array}$ & $\begin{array}{l}\text { Fever, headache (malaria), } \\
\text { asthma, cough }\end{array}$ & FEV/MAL/RESP & Sap & S & $\mathrm{O}$ \\
\hline GW 04/04 & Sterculia shillinglawii F. Muell. & Malvaceae & $\begin{array}{l}\text { Huasiva or } \\
\text { Chosembi }\end{array}$ & Enlarged spleen, pigbel & ORG/GAST & L | Sap & $D \mid S$ & $\mathrm{O}$ \\
\hline DK 09/05 & $\begin{array}{l}\text { Syzygium malaccense (L.) Merr. \& L.M. Perry } \\
{[7,8,34,39,42,47,56,67]}\end{array}$ & Myrtaceae & Gwangolik & Fever & FEV & L & $\mathrm{D}$ & T \\
\hline DK 10/05 & $\begin{array}{l}\text { Syzygium malaccense (L.) Merr. \& L.M. Perry } \\
{[7,8,34,39,42,47,56,67]}\end{array}$ & Myrtaceae & Turukirmba & Fever & FEV & L & $\mathrm{D}$ & T \\
\hline MS 43/04 & $\begin{array}{l}\text { Syzygium malaccense (L.) Merr. \& L.M. Perry } \\
{[7,8,34,39,42,47,56,67]}\end{array}$ & Myrtaceae & Duokuma & Epigastric pain & GAST & L & $\mathrm{H}$ & $\mathrm{T}$ \\
\hline BK 048/06 & Syzygium sp. $[8,9,33,43,45]$ & Myrtaceae & Kaviak & Cough with itchy throat & RESP & $y L$ & $\mathrm{D}$ & $\mathrm{O}$ \\
\hline BK 054/06 & Tabernaemontana pandacaqui Lam $[34,38,46]$ & Apocynaceae & Karaban & Grille & SKIN & Fruit & S & T \\
\hline GW 76/04 & Tabernaemontana sp. & Apocynaceae & Raviapari & Determine baby girl & REP & Root & M & $\mathrm{O}$ \\
\hline GW 82/04 & Tinospora arfakiana Becc. & Menispermaceae & Saihuna & Cough, grille & RESP/SKIN & L & $D \mid S$ & $\mathrm{O} \mid \mathrm{T}$ \\
\hline MS 65/04 & Tinospora sp. & Menispermaceae & Tifoniak kuriri & Asthma, cough & RESP & L & S & O \\
\hline GW 57/04 & Tylophora sp. & Asclepiadaceae & Yousa & Recovery from illness & NUT & Root & $\mathrm{D}$ & $\mathrm{O}$ \\
\hline BK 005/06 & Uncaria lanosa var. appendiculata (Benth.) Ridsdale & Rubiaceae & Marangi & $\begin{array}{l}\text { Fever, headache, malaria, cough, } \\
\text { malnutrition }\end{array}$ & $\begin{array}{l}\text { MAL/FEV/NUT/ } \\
\text { HEAD/RESP }\end{array}$ & Sap & s & O \\
\hline MS 82/04 & Uncaria lanosa var. appendiculata (Benth.) Ridsdale & Rubiaceae & Mewow & $\begin{array}{l}\text { Severe fever, chronic diarrhoea with } \\
\text { blood, loss of weight. }\end{array}$ & FEV/GAST & Sap & s & $\mathrm{O}$ \\
\hline DK 29/05 & Uncaria orientalis Guillaumin & Rubiaceae & Marange & Shortness of breath & RESP & Sap & S & $\mathrm{O}$ \\
\hline GW 85/04 & Uncaria sp. $[8,9,33,35,39]$ & Rubiaceae & Trakiau kakoin & Headache, migraine & HEAD & Sap & S & $\mathrm{O}$ \\
\hline GW 72/04 & Ursi sp. & Fabaceae & Swamareng & Determine baby boy & REP & Root & S & $\mathrm{O}$ \\
\hline
\end{tabular}


Table 1 Plants reported as medicinally used in 4 study areas in East Sepik Province (Continued)

\begin{tabular}{|c|c|c|c|c|c|c|c|c|}
\hline GW 77/04 & $\begin{array}{l}\text { Urticastrum decumanum (Roxb.) Kuntze } \\
{[9,32,34-37,39,42-45,49,51,53,59,63,65,66,} \\
69,71,72,74-77]\end{array}$ & Urticaceae & Purkumb & $\begin{array}{l}\text { B body, muscle, joint pains, } \\
\text { pneumonia }\end{array}$ & PAIN/RESP & L & R & $\mathrm{O} \mid \mathrm{T}$ \\
\hline MS 62/04 & $\begin{array}{l}\text { Urticastrum decumanum (Roxb.) Kuntze }[9,32, \\
\text { 34-37, 39, 42-45, 49, 51, 53, 59, 63, 65, 66 69, 71, } \\
72,74-77]\end{array}$ & Urticaceae & Chipia & Abortion & REP & L & $\mathrm{D}$ & O \\
\hline BK 012/06 & Vanilla sp. [65] & Orchidaceae & $\begin{array}{l}\text { Dunauru } \\
\text { banguwi }\end{array}$ & Prevent miscarriage & REP & Sap & S & O \\
\hline MS 13/04 & Villebrunea sp. & Urticaceae & Wurarian & $\begin{array}{l}\text { Very high fever, headache, } \\
\text { swollen bodies }\end{array}$ & $\begin{array}{l}\text { FEV/HEAD/ } \\
\text { SWELL }\end{array}$ & Sap & S & O \\
\hline MS 86/04 & Virola surinamensis (Rol. ex Rottb.) Warb [33] & Myristicaceae & Sukuai & Sore in the baby's mouth & CHILD & L & MS & T \\
\hline GW 73/04 & $\begin{array}{l}\text { Wedelia biflora (L.) DC. }[34,38,39,42,43,46,48 \text {, } \\
52,56,57,59,62,63,67,68,70]\end{array}$ & Asteraceae & Bambawhoo & $\begin{array}{l}\text { Cough, diarrhoea, women's } \\
\text { bleeding disorders }\end{array}$ & RESP/GAST/REP & L & $\mathrm{D}$ & O \\
\hline BK 019/06 & $\begin{array}{l}\text { Wedelia sp. }[34,38,39,42,43,46,48,52,56,57 \text {, } \\
59,62,63,67,68,70]\end{array}$ & Asteraceae & Pava & Running nose, cough,asthma & RESP & L & V & I \\
\hline MS 72/04 & $\begin{array}{l}\text { Wedelia sp. }[34,38,39,42,43,46,48,52,56,57 \text {, } \\
59,62,63,67,68,70]\end{array}$ & Asteraceae & Kiskiash & Toothache & DENT & yShoot & M & $\mathrm{O}$ \\
\hline BK 030/06 & $\begin{array}{l}\text { Zingiber officinale Roscoe }[8,9,34,36,39,42,53 \text {, } \\
56,60,63,65-67,71,73,77]\end{array}$ & Zingiberaceae & Kambei laki & Ssnake bites & BITE & L & R & $O \& T$ \\
\hline DK 07/05 & $\begin{array}{l}\text { Zingiber officinale Roscoe }[8,9,34,36,39,42,53 \text {, } \\
56,60,63,65-67,71,73,77]\end{array}$ & Zingiberaceae & Nikirkuasa & Malaria & MAL & Whole & $\mathrm{D}$ & T \\
\hline DK 39/05 & $\begin{array}{l}\text { Zingiber officinale Roscoe }[8,9,34,36,39,42,53 \text {, } \\
56,60,63,65-67,71,73,77]\end{array}$ & Zingiberaceae & Huaukuasa & Malaria & MAL & Whole & $\mathrm{D}$ & O \\
\hline MS 45/04 & $\begin{array}{l}\text { Zingiber officinale Roscoe }[8,9,34,36,39,42,53, \\
56,60,63,65-67,71,73,77]\end{array}$ & Zingiberaceae & Leai & Epigastric pain, vomiting, diarrhoea & GAST & Root & MS & $O \& T$ \\
\hline
\end{tabular}

Ailment treated (Ailmentcode) as follows: BITE $=$ insect or snake bite; BLOOD $=$ hematological issues including coagulation; $B O N E=$ bone related injury or disease; $B$ URN $=$ burns; $C A N C=$ cancer; $C H I L D=$ childhood disease; $C V$ = Cardiovascular; DENT = dental disease; FEV = fever; GAST = gasteroenterological disease; HEAD = headache; INF = infection; INSECTICIDE = delousing; MAGIC = disease of unidentified etiology ('magical poisoning'); MAINT = health promotion, including failure to thrive; $M A L=$ Malaria; $N U T=$ nutritional supplement; $O C C=$ ocular diseases; $\mathrm{ORG}=$ diseases thought to affect one particular organ; OTHER = unclear disease syndrome; PAIN = physical pain; POIS = envenomation or poisoning; sometimes this includes transnatural causation; PSYCH = psychiatric diseases or syndromes; REP = reproductive diseases including childbirth related syndrome; PAIN = physical pain; $\mathrm{POIS}=$ envenomation or poisoning; sometimes this includes transnatural causation; $\mathrm{PSYCH}=$ psychiatric diseases or syndromes; REP $=$ reproductive diseases including childbirth related
issues; RESP = respiratory diseases; SKIN = dermal related diseases; often includes infectious disease; SWELL = swelling of whole body or part of the body; URINE = urinary conditions; WOUND = wound related diseases or syndromes

Route of Administration codes (RouteCode) as follows: $\mathrm{O}=$ oral; $\mathrm{T}=$ topical; $\mathrm{I}=$ inhalation; P_to_Plant $=$ patient to plant transfer of blood

Mode of preparation codes (PrepCode) as follows: $B=$ burned (smoke generation), $C=$ cooked; $D=$ decoction, $\mathrm{H}=$ heated, $\mathrm{HR}=$ heated then rubbed, $\mathrm{M}=$ masticated, $\mathrm{MAG}=$ magical, $\mathrm{MS}=$ masticated then spit on affected area(s), $R=$ raw; $S=$ succus (crushed), $V=$ vapor

Plant part utilized codes (PartCode) as follows: $\mathrm{R}=$ Rhizome, $\mathrm{L}=\mathrm{Leaf}, \mathrm{L}=$ = young leaf, $\mathrm{B}=$ Bark, $\mathrm{y}$ Shoot $=$ young shoo 
Table 2 Plants not shared between the four study areas in East Sepik Province

\begin{tabular}{|c|c|c|c|}
\hline BK & DK & GW & MS \\
\hline $\begin{array}{l}\text { Albizia saman (Jacq.) Merr. } \\
\text { (BK 058/06) }\end{array}$ & $\begin{array}{l}\text { Ageratum conyzoides (L.) L. (DK 38/ } \\
\text { 05) }\end{array}$ & $\begin{array}{l}\text { Albizia procera (Roxb.) Benth. } \\
\text { (GW 09/04) }\end{array}$ & $\begin{array}{l}\text { Abelmoschus manihot (L.) Medik. } \\
\text { (MS 02/04) }\end{array}$ \\
\hline $\begin{array}{l}\text { Cascabela thevetia (L.) Lippold } \\
\text { (BK 028/06) }\end{array}$ & $\begin{array}{l}\text { Angiopteris evecta (G. Forst.) Hoffm. } \\
\text { (DK 53/05) }\end{array}$ & Bidens pilosa L. (GW 40/04) & $\begin{array}{l}\text { Artocarpus altilis (Parkinson ex } \\
\text { F.A. Zorn) Fosberg (MS 23/04) }\end{array}$ \\
\hline $\begin{array}{l}\text { Murraya paniculata } \\
\text { (BK 003/06) }\end{array}$ & Areca catechu L. (DK 02/05) & $\begin{array}{l}\text { Cerbera floribunda K. Schum. (GW 12/ } \\
\text { 04) }\end{array}$ & $\begin{array}{l}\text { Barringtonia asiatica (L.) Kurz } \\
\text { (MS 27/04) }\end{array}$ \\
\hline $\begin{array}{l}\text { Premna serratifolia L. } \\
\text { (BK 052/06) }\end{array}$ & Asplenium nidus L. (DK 21/05) & Clitoria terneata L. (GW 91/04) & $\begin{array}{l}\text { Callicarpa longifolia Lam. } \\
\text { (MS 85/04) }\end{array}$ \\
\hline \multirow[t]{13}{*}{$\begin{array}{l}\text { Tabernaemontana pandacaqui Lam } \\
\text { (BK 054/06) }\end{array}$} & Bixa orellana L. (DK 11/05) & $\begin{array}{l}\text { Gymnostoma papuana (S. Moore) } \\
\text { L.A.S. Johnson (GW 11/04) }\end{array}$ & $\begin{array}{l}\text { Calophyllum inophyllum L. } \\
\text { (MS 20/04) }\end{array}$ \\
\hline & Capsicum annuum L. (DK 15/05) & $\begin{array}{l}\text { Hemigraphis reptans (G. Forst.) T. } \\
\text { Anders. ex Hemsl. (GW 70/04) }\end{array}$ & $\begin{array}{l}\text { Calotropis gigantea (L.) Dryand } \\
\text { (MS 32/04) }\end{array}$ \\
\hline & Carica papaya L. (DK 34/05) & $\begin{array}{l}\text { Hydriastele costata F.M. Bailey (GW } \\
83 / 04 \text { ) }\end{array}$ & $\begin{array}{l}\text { Caryota rumphiana Mart. } \\
\text { (MS 69/04) }\end{array}$ \\
\hline & Caryota mitis Lour. (DK 26/05) & $\begin{array}{l}\text { Maclura cochinchinensis (Lour.) } \\
\text { Corner (GW 46/04) }\end{array}$ & Casuarina equisetifolia L. (MS 28/04) \\
\hline & $\begin{array}{l}\text { Cheilocostus speciosus (J. König) C. } \\
\text { Specht (DK 20/05) }\end{array}$ & Mangifera indica L. (GW 93/04) & $\begin{array}{l}\text { Chrysopogon aciculatus (Retz). } \\
\text { Trin (MS 50/04) }\end{array}$ \\
\hline & $\begin{array}{l}\text { Dendrocnide cordata (Warb. ex H.J.P. } \\
\text { Winkl.) Chew (DK 35/05) }\end{array}$ & $\begin{array}{l}\text { Neonauclea purpurea (Roxb.) Merr. } \\
\text { (GW 10/04) }\end{array}$ & Cocos nucifera L. (MS 78/04) \\
\hline & $\begin{array}{l}\text { Homalium foetidum (Roxb.) Benth. } \\
\text { (DK 42/05) }\end{array}$ & $\begin{array}{l}\text { Pisonia longirostris Teijsm. \& Binn. } \\
\text { (GW 32/04) }\end{array}$ & $\begin{array}{l}\text { Dendrocnide latifolia (Gaudich.) } \\
\text { Chew (MS 33/04) }\end{array}$ \\
\hline & Manihot esculenta Crantz (DK 51/05) & Solanum torvum Sw. (GW 26/04 & $\begin{array}{l}\text { Euphorbia tithymaloides (L.) } \\
\text { (MS 79/04) }\end{array}$ \\
\hline & Metroxylon sagu Rottb. (DK 30/05) & $\begin{array}{l}\text { Sterculia shillinglawii F. Muell. (GW } \\
\text { 04/04) }\end{array}$ & Ocimum basilicum L. (MS 08/04) \\
\hline & $\begin{array}{l}\text { Piscidia grandifolia (Donn. Sm.) I.M. } \\
\text { Johnst. (DK 31/05) }\end{array}$ & & $\begin{array}{l}\text { Pandanus dubius Spreng. (MS 30/ } \\
\text { 04) }\end{array}$ \\
\hline & $\begin{array}{l}\text { Planchonia papuana R. Knuth } \\
\text { (DK 45/05) }\end{array}$ & & Scaevola sericea Vahl (MS 63/04) \\
\hline & $\begin{array}{l}\text { Riedelia corallina (K. Schum.) Valeton } \\
\text { (DK 18/05) }\end{array}$ & & $\begin{array}{l}\text { Schismatoglottis calyptrata (Roxb.) } \\
\text { Zoll. \& Moritzi (MS 83/04) }\end{array}$ \\
\hline & & & Sida rhombifolia L. (MS 76/04) \\
\hline \multicolumn{4}{|l|}{ Identified to Genus only (Voucher) } \\
\hline Christia sp. (BK 008/06) & Cinnamonum sp. (DK 54/05) & Aglaia sp. (GW 56/04) & Archidendron sp. (MS 01/04) \\
\hline Clematis sp. (BK 049/06) & & Asclepias sp. (GW 79/04) & Davallia sp. (MS 70/04) \\
\hline Neonauclea sp. (BK 061/06) & & Cissus sp. (GW 59/04) & Dillenia sp. (MS 81/04) \\
\hline Phrynium sp. (BK 014/06) & & Clerodendrum sp. (GW 87/04) & Graptophyllum sp. (MS 14/04) \\
\hline \multirow[t]{11}{*}{ Vanilla sp. (BK 012/06) } & & Desmodium sp. (GW 101/04) & Homalanthus sp. (MS 05/04) \\
\hline & & Mitracarpus sp. (GW 20/04) & Marattia sp. (MS 16/04) \\
\hline & & Papuechites sp. (GW 65/04) & Melastoma sp. (MS 36/04) \\
\hline & & Parsonia sp. (GW 29/04) & Villebrunea sp. (MS 13/04) \\
\hline & & Pouteria sp. (GW 41/04) & \\
\hline & & Semecarpus sp. (GW 55/04) & \\
\hline & & Smilax sp. (GW 99/04) & \\
\hline & & Spathiphyllum sp. (GW 33/04) & \\
\hline & & Tabernaemontana sp. (GW 76/04) & \\
\hline & & Tylophora sp. (GW 57/04) & \\
\hline & & Ursi sp. (GW 72/04) & \\
\hline
\end{tabular}




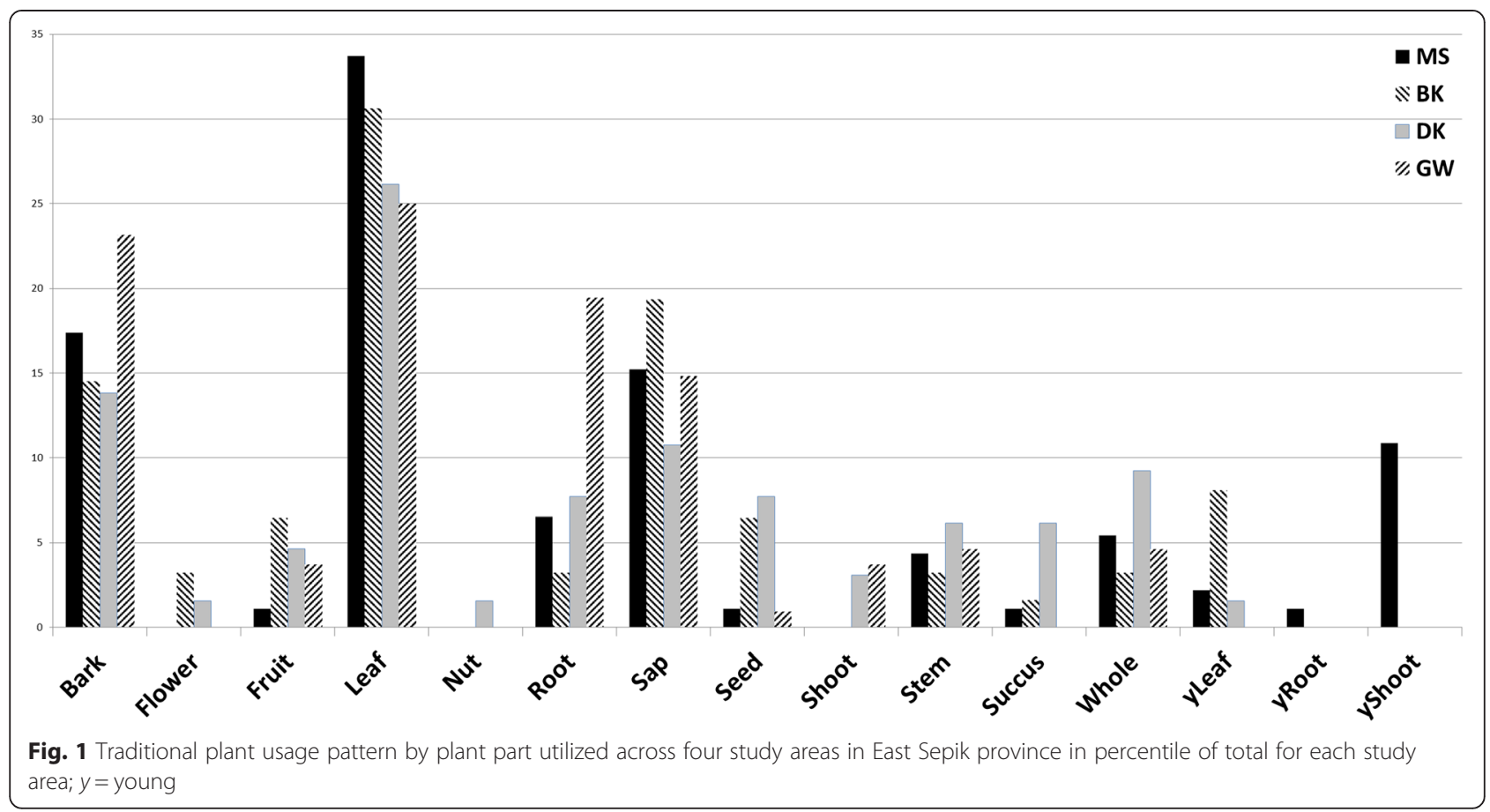

infections (e.g., "Grille") and ectoparasitism (e.g., scabies); the category "REP" contains all sort of reproductive conditions, e.g., impotence, abortion, menstrual syndromes, contraception and fertility, etc. The exception to this method of categorization is malaria, which is generally well recognized throughout the Sepik. Overall, skin conditions were most frequently treated (73 instances), with respiratory conditions (60 instances), fever (39 instances), gastrointestinal conditions (36 instances) and malaria (29 instances) rounding out the top five conditions. The top five conditions in the respective reports were: for MS (fever-19, skin-18, headache-16, respiratory and gastrointestinal-12 reports each); BK (skin-22, respiratory-15, gastrointestinal

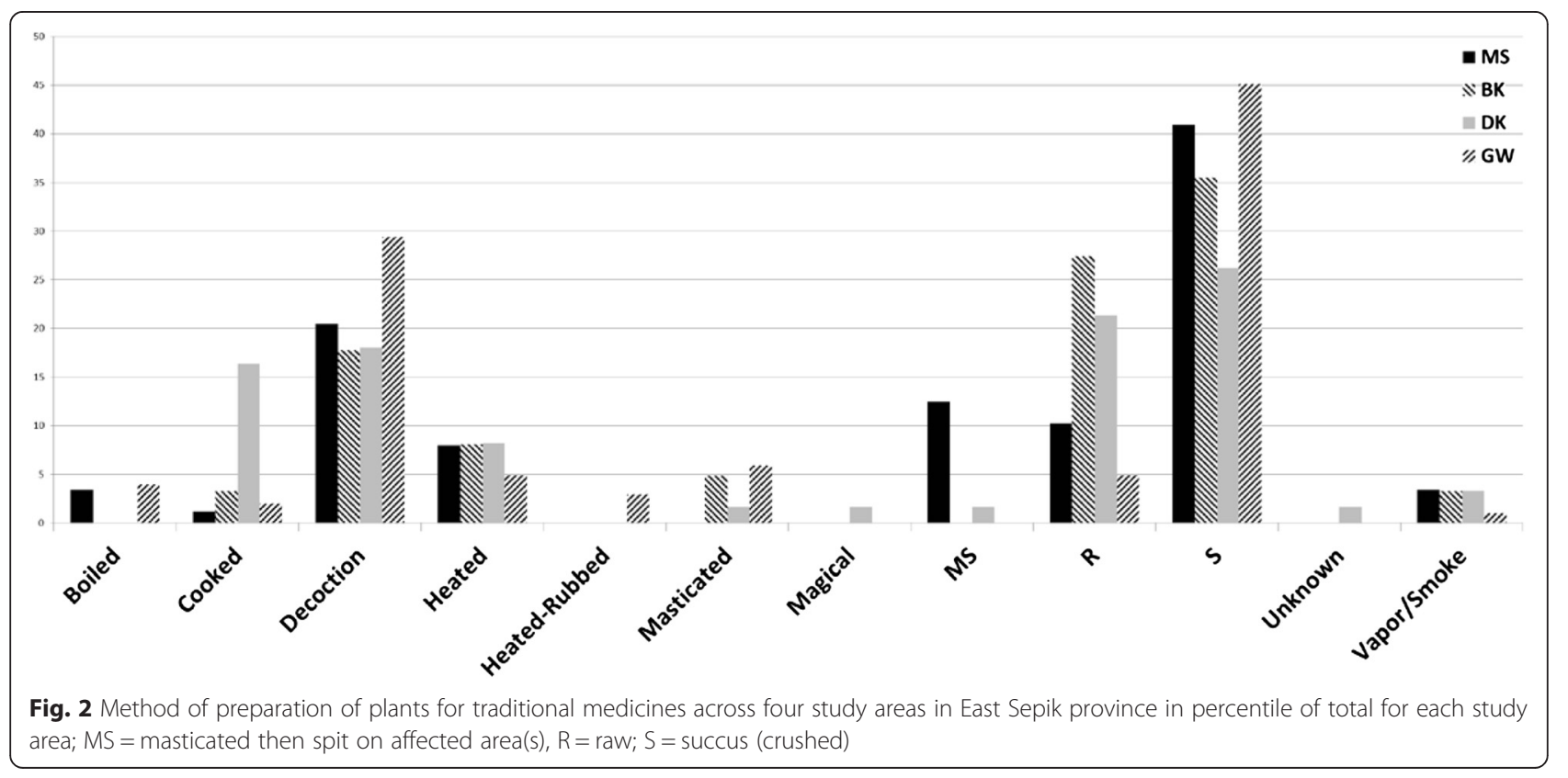




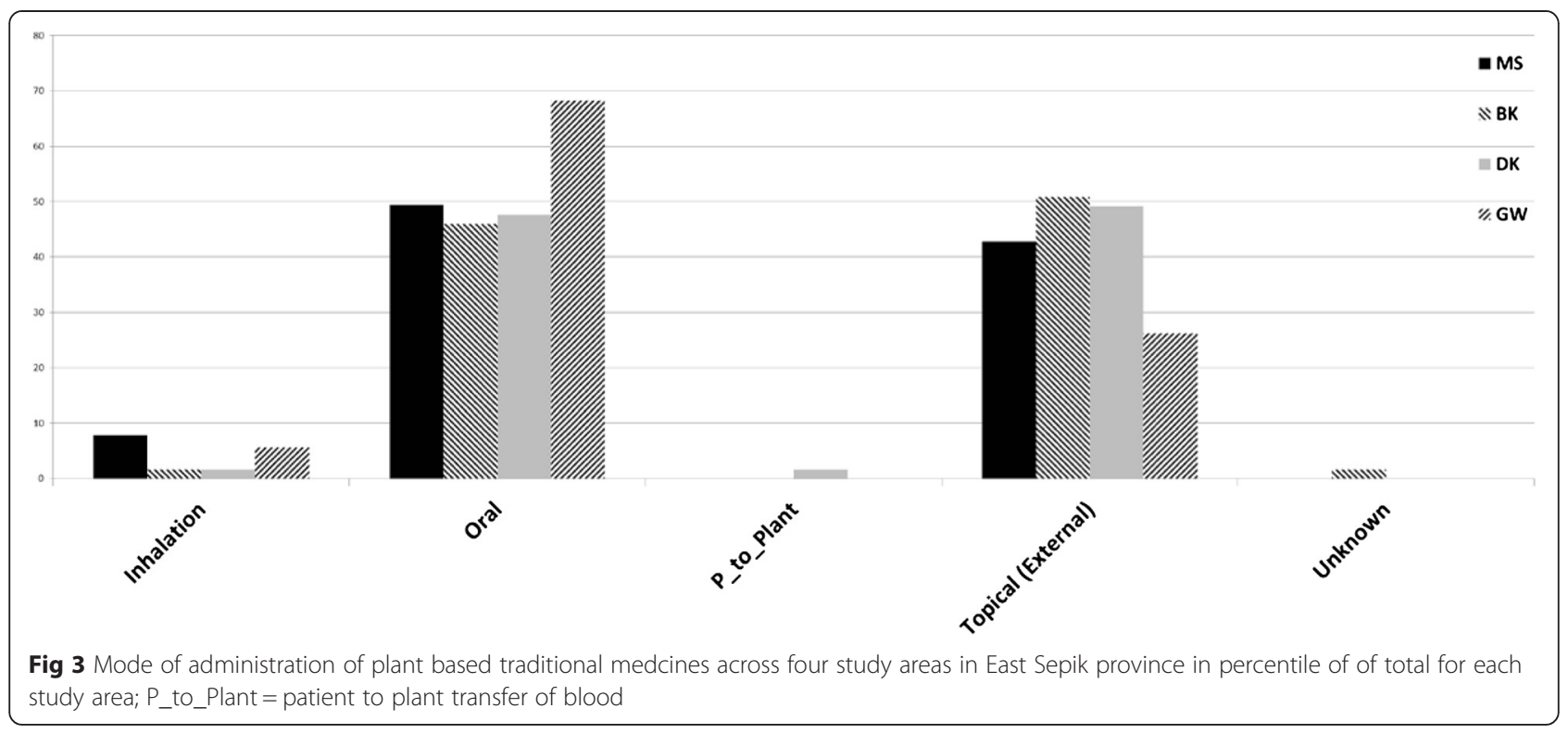

conditions -7 wounds -6 , and pain -5 instances): DK (skin-16, respiratory-8, malaria and wounds-5 instances each, and fever-4 instances): GW (respiratory-25, skin and malaria-17 each, gastrointestinal conditions and fever-14 instances each). The relative frequencies of ailments/conditions are presented in Fig. 4. Outlier conditions, those reported once and not reported in the other areas were urinary conditions (incontinence, URINE; and delousing, INSECTICIDE) from the MS report; use for burn conditions (BURN), magical poisoning

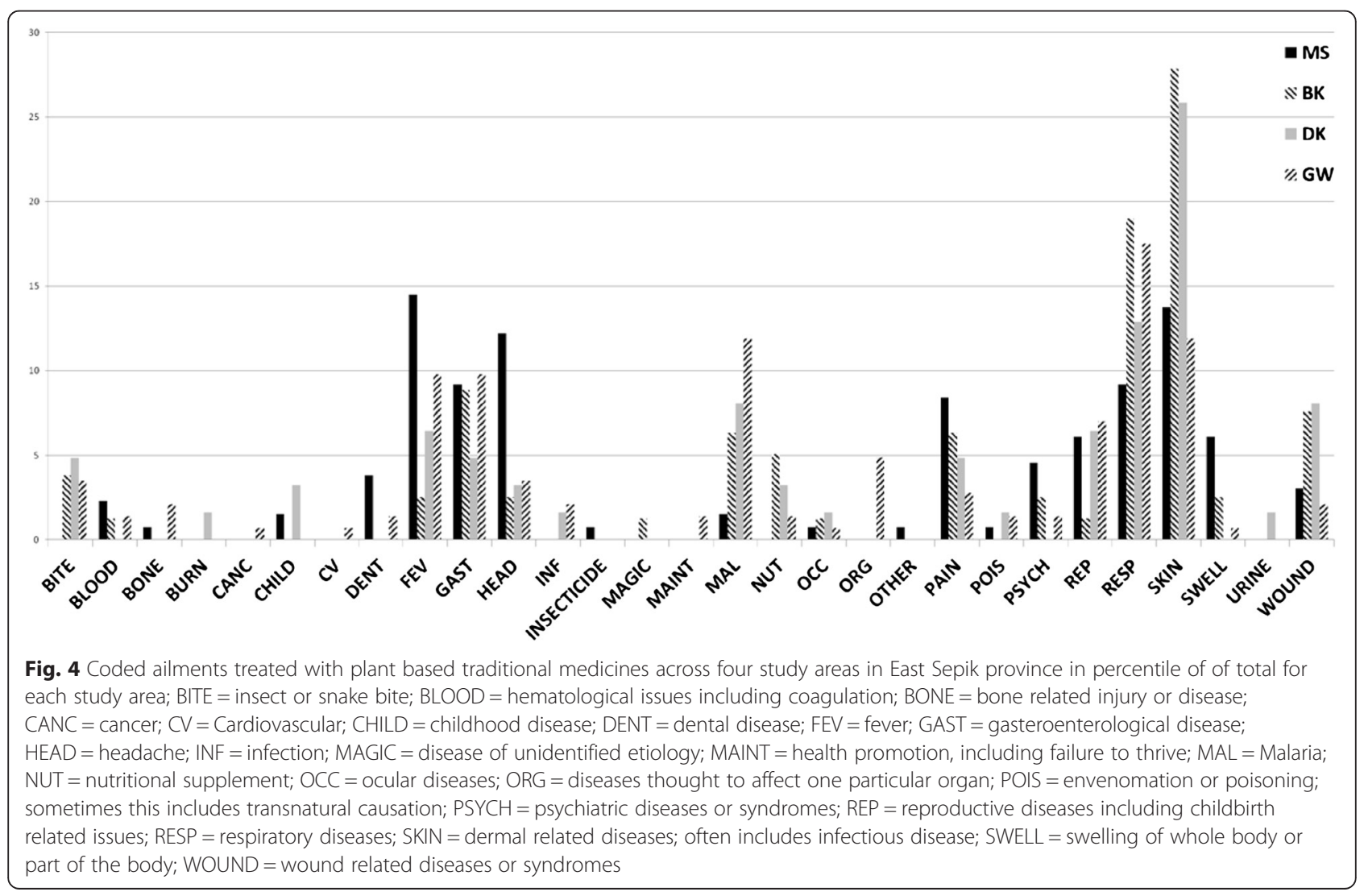


(MAGIC) and child health improvement (CHILD) from the DK area; and cancer (CANC) and cardiovascular condition (CV) from the GW area.

\section{Most common families of plants used by healers interviewed}

By far the most common genus was Ficus (11), followed by Euphorbia (7), Piper (6), Plectranthus (6), Cassia (5), Passiflora (5), and 4 instances each of: Acalypha, Alpinia, Alstonia, Calamus, Crinum, Gnetum, Laportea, Merremia, Mucuna, Phyllanthus, Syzygium, Uncaria, and Zingiber.

\section{Lesser known medicinal plant species of East Sepik}

Those plants identified to the species level and not found in the Bougainville and Eastern Highlands reports were matched against our medicinal plants of PNG reference database, consisting of historical reports largely by Holdsworth and associates. The following plants were not described in the literature which the database encompasses: Averrhoa carambola L. (BK 039/06 \& DK 01/05), Campnosperma brevipetiolatum Volkens Volkens. (DK 56/05), Capsicum annuum L. (DK 15/05), Caryota mitis Lour. (DK 26/05), Cascabela thevetia (L.) Lippold (BK 028/06), Chrysopogon aciculatus (Retz). Trin (MS 50/ 04), Clitoria ternatea L. (GW 91/04), Curcuma longa L. (BK 029/06), Cycas rumphii Miq. (BK 002/06), Endospermum labios Schodde (DK 40/05), Endospermum formicarium Becc. (GW 28/04), Endospermum medullosum L.S.Sm. (MS 89/04), Erythrina merrilliana Krukoff (GW 18/04 \& MS 42/04), Hydriastele costata F.M. Bailey (GW 83/04), Intsia bijuga (Colebr.) Kuntze (DK 33/05 \& GW 08/04 \& MS 46/04), Millettia pinnata (L.) Panigrahi (GW 30/04), Planchonia papuana R. Knuth (DK 45/05), Riedelia corallina (K. Schum.) (DK 18/05), Schismatoglottis calyptrata (Roxb.) Zoll. \& Moritzi (MS 83/04), Sterculia shillinglawii F. Muell. (GW 04/04), and Tinospora arfakiana Becc. (GW 82/04).

Capsicum annuum L. and Curcuma longa L. are commonly grown in many gardens across PNG, yet it was surprising to note the paucity of medicinal uses previously reported for PNG. Ipomoea pes-caprae (L.) R. Br. (BK 020/06 \& MS 26/04) also did not appear to be part of the older literature, however, it was recently found to be used in the New Britain Province where the leaves are rubbed onto the skin affected by jelly fish stings [12]. The sap is used in the BK area for respiratory ailments, and the succus from the leaves is reported by MS to be used in Kairiru for fever/pain via oral consumption.

\section{Comparing East Sepik with Eastern highlands and Bougainville provinces}

The combined dataset of the East Sepik, Eastern Highlands and Bougainville reports encompasses 276 plant genera, of which only 22 were reported in common from our other published data sets; Bougainville 112 genera, Eastern highlands 121, and East Sepik 154 genera (see Fig. 5). The frequency of shared genera is given in Table 3. The plant genera with the highest common use citations $(>=10)$ are Ficus sp. 29, Alpinia sp. 16, Piper sp. 15, Syzygium sp. 12 and Alstonia sp.11. The predominance of Ficus sp. is not surprising since Ficus represents a very large genus in PNG [13].

\section{Regional utilization of plants}

Comparison of plants used medicinally in our published data sets to a general list of plants from the same regions allowed for an analysis of utilization preferences. Medicinally over- and under-represented plant families are given in Table 4, while medical plant utilization is given in Table 5. Comparison shows that the number of plant families significantly underutilized, when compared against the regional flora, breaks down as follows: in East Sepik (ES) province Poaceae are underutilized, while in the Eastern Highlands (EH) and Bougainville (BV) Orchidaceae are underutilized.

The number of plants overutilized varies (ES: $n=15$; $\mathrm{EH}: n=25$ and BV: $n=12$ ) but is relatively stable as percentage of plants found in the regional database at 0.66, 0.7 and $0.78 \%$ for ES, EH and BV, respectively. East

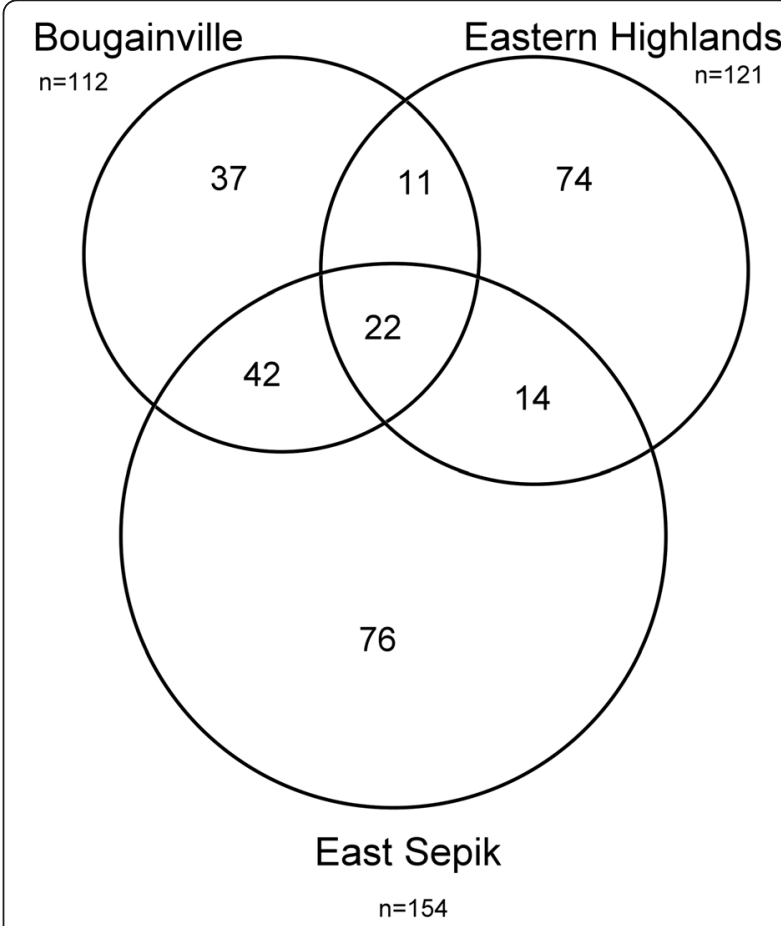

Fig. 5 Venn diagram showing the total number ( $n$ ) and overlap of plant genera utilized medicinally between Bougainville, East Sepik, and Eastern Highlands provinces 
Table 3 Plant Genera in common utilized in Bougainville, Eastern Highlands and East Sepik Provinces

\begin{tabular}{lllll}
\hline Genus & Bougainville & Eastern Highlands & East Sepik & Total \\
\hline Ageratum & 2 & 1 & 1 & 4 \\
Alpinia & 7 & 5 & 4 & 16 \\
Alstonia & 4 & 3 & 4 & 11 \\
Aristolochia & 1 & 1 & 2 & 4 \\
Barringtonia & 2 & 1 & 1 & 4 \\
Ficus & 11 & 7 & 11 & 29 \\
Graptophyllum & 1 & 1 & 1 & 3 \\
Hemigraphis & 1 & 2 & 1 & 4 \\
Leucosyke & 1 & 1 & 1 & 3 \\
Litsea & 1 & 1 & 2 & 4 \\
Melastoma & 1 & 2 & 1 & 4 \\
Mucuna & 3 & 1 & 5 & 9 \\
Musa & 2 & 1 & 2 & 5 \\
Piper & 4 & 5 & 6 & 15 \\
Plectranthus & 2 & 1 & 6 & 9 \\
Psidium & 2 & 1 & 2 & 5 \\
Sida & 1 & 1 & 1 & 3 \\
Smilax & 1 & 3 & 1 & 5 \\
Syzygium & 4 & 4 & 4 & 12 \\
Uncaria & 2 & 1 & 5 \\
Zingiber & 1 & 2 & 2 & 7 \\
\hline & & & 4 &
\end{tabular}

Sepik shares overutilization of Fabaceae, Gnetaceae and Zingiberaceae with Bougainville and overutilization of Asteraceae and Lamiaceae with Eastern Highlands, while Eastern Highlands and Bougainville share no overutilized plant families.

When the UPNG Traditional Medicines Database was used to assess utilization, the underrepresented plant families were the Verbenaceae in East Sepik and the Euphorbiaceae in the Eastern Highlands. No plant family met the $p=0.05$ criterion in Bougainville, however, Euphorbiaceae was the top ranked underutilized plant family (data not shown). The number of overutilized plants is varied (ES: $n=4 ; \mathrm{EH}: n=17 ; \mathrm{BV}: n=12$ ). Among the overused plant families East Sepik shared the Arecaceae with Bougainville. Several plant families reappear in this analysis, e.g., the Asteraceae and Winteraceae from the Eastern Highlands province and the Gnetaceae and Zingiberaceae in Bougainville. The statistical requirements of the comparison method resulted in some plant families appearing in the overutilization category represent a single report from the region for that plant family. This could not be avoided since the East Sepik reports are included in the UPNG Traditional Medicines Database total. As the PNG Medicinal Plant
Database database grows in the future the stringency of the analysis will improve.

Traditional inspection of the information gathered yielded information about plants not widely used, poorly annotated or used for different ailments than those in locales where use of the plant is more common. Plants without annotation in the recent PNG Medicinal Plant Literature include:

Alocasia cucullata (Lour.) G. Don surprisingly did not yield any crossrefernces in the PNG database, even when using synonyms. It is used in Chinese medicine for snakebite, abscesses, rheumatism, and arthritis [14] and has recently been identified as containing anticancer compounds $[15,16]$.

Averrhoa carambola L. (starfruit) fruit is used for cuts and asthma in PNG, and also widely used throughout the world for a variety of ailments, seemingly only in India as antihemmoraghic [17].

Caryota mitis Lour. has no further medicinal annotation for use in PNG, but is used several Asian countries for a variety of ailments, e.g., against hemorrhoids, male sexual dysfunction, and rheumathoid arthritis in Bangladesh [17].

Chrysopogon aciculatus (Retz). Trin is used in the East Sepik for swelling. The plant is used in Ayurveda as a diuretic $[17,18]$.

Clitoria ternatea L.is used for infertility in PNG and similarly in Ayurveda, where fresh root juice in fresh goat milk is used for pregnancy [18], however, the plant is used for a dizzying array of conditions and ascribed activities [17].

Endospermum medullosum L.S.Sm. has been described previously as used against rheumatism [18], perhaps similar to the use against general body pain in the East Sepik.

Used as a contraceptive in the East Sepik, Erythrina merrilliana Krukoff reveals a dearth of information regarding medicinal uses. The plant is however known to produce toxic alkaloids [19].

Gnetum gnemonoides Brongn. yielded very little information as to medicinal use, but has been described to contain a variety of stilbenes [20].

Hemigraphis reptans (G. Forst.) T. Anderson ex Hemsl. is used in the East Sepik as the whole plant to treat centipede bite. The root is expressed into water to facilitate birth (speeding up delivery) on Vanuatu [21].

No medicinal use annotation was found for Hydriastele costata F.M. Bailey and therefore it may present one of the plants which is used very rarely for that purpose.

Intsia bijuga (Colebr.) Kuntze has annotations as a detoxicant and against diarrhea, toothache, adenopathy and swelling [22].

Macaranga clavata Warb. is used in East Sepik for skin infections, but has no recent mention in the literature for 
Table 4 Overrepresented and underespresented plants for each province when compared to the regional plant diversity as recorded in the PNG Plant Database

\begin{tabular}{|c|c|c|c|c|c|}
\hline \multicolumn{6}{|c|}{ East Sepik (ES Reports vs PNG PlantDB (ES total flora) } \\
\hline & $\begin{array}{l}\text { \# in PNG } \\
\text { PlantDB }\end{array}$ & $\begin{array}{l}\text { \# in ES Medical Plants } \\
\text { Reports }\end{array}$ & $\begin{array}{l}\text { Inferior Credible } \\
\text { Interval }\end{array}$ & $\begin{array}{l}\text { Superior Credible } \\
\text { Interval }\end{array}$ & $\begin{array}{l}\text { Difference to interv } \\
\text { (margin) }\end{array}$ \\
\hline Total & 2258 & 207 & 0.080 & 0.104 & - \\
\hline \multicolumn{6}{|c|}{ Overrepresented Families } \\
\hline Araceae & 13 & 7 & 0.289 & 0.770 & 0.184 \\
\hline Zingiberaceae & 14 & 6 & 0.213 & 0.677 & 0.108 \\
\hline Marantaceae & 3 & 2 & 0.194 & 0.932 & 0.090 \\
\hline Solanaceae & 9 & 4 & 0.187 & 0.738 & 0.083 \\
\hline Euphorbiaceae & 83 & 22 & 0.182 & 0.369 & 0.078 \\
\hline Convolvulaceae & 13 & 5 & 0.177 & 0.649 & 0.072 \\
\hline Datiscaceae & 1 & 1 & 0.158 & 0.987 & 0.054 \\
\hline Fabaceae & 82 & 19 & 0.154 & 0.334 & 0.050 \\
\hline Gnetaceae & 4 & 2 & 0.147 & 0.853 & 0.042 \\
\hline Davalliaceae & 8 & 3 & 0.137 & 0.701 & 0.033 \\
\hline Lamiaceae & 42 & 10 & 0.135 & 0.386 & 0.031 \\
\hline Anacardiaceae & 18 & 5 & 0.126 & 0.512 & 0.021 \\
\hline Asteraceae & 19 & 5 & 0.119 & 0.491 & 0.015 \\
\hline \multicolumn{6}{|l|}{ Menispermaceae } \\
\hline Piperaceae & 15 & 4 & 0.110 & 0.524 & 0.006 \\
\hline \multicolumn{6}{|c|}{ Underrepresented Families } \\
\hline Poaceae & 106 & 3 & 0.010 & 0.080 & 0.028 \\
\hline
\end{tabular}

Eastern Highlands (EH) vs PNG PlantDB (EH Total Flora)

\begin{tabular}{|c|c|c|c|c|c|}
\hline & $\begin{array}{l}\text { \# in PNG } \\
\text { PlantDB }\end{array}$ & $\begin{array}{l}\text { \# in EH Medical Plants } \\
\text { Reports }\end{array}$ & $\begin{array}{l}\text { Inferior Credible } \\
\text { Interval }\end{array}$ & $\begin{array}{l}\text { Superior Credible } \\
\text { Interval }\end{array}$ & $\begin{array}{l}\text { Difference to interval } \\
\text { (margin) }\end{array}$ \\
\hline Total & 3549 & 156 & 0.038 & 0.051 & - \\
\hline \multicolumn{6}{|c|}{ Overrepresented Families } \\
\hline Ebenaceae & 2 & 2 & 0.292 & 0.992 & 0.241 \\
\hline Winteraceae & 2 & 2 & 0.292 & 0.992 & 0.241 \\
\hline Acanthaceae & 12 & 5 & 0.192 & 0.684 & 0.141 \\
\hline Hypoxidaceae & 1 & 1 & 0.158 & 0.987 & 0.107 \\
\hline Smilacaceae & 7 & 3 & 0.157 & 0.755 & 0.106 \\
\hline Plantaginaceae & 5 & 2 & 0.118 & 0.777 & 0.067 \\
\hline Lamiaceae & 21 & 5 & 0.107 & 0.454 & 0.056 \\
\hline Araliaceae & 17 & 4 & 0.097 & 0.476 & 0.046 \\
\hline Commelinaceae & 2 & 1 & 0.094 & 0.906 & 0.043 \\
\hline Elaeagnaceae & 2 & 1 & 0.094 & 0.906 & 0.043 \\
\hline Actinidiaceae & 14 & 3 & 0.078 & 0.481 & 0.027 \\
\hline Asteraceae & 103 & 13 & 0.076 & 0.204 & 0.024 \\
\hline Bignoniaceae & 3 & 1 & 0.068 & 0.806 & 0.016 \\
\hline Casuarinaceae & 3 & 1 & 0.068 & 0.806 & 0.016 \\
\hline Lecythidaceae & 3 & 1 & 0.068 & 0.806 & 0.016 \\
\hline Symplocaceae & 3 & 1 & 0.068 & 0.806 & 0.016 \\
\hline Onagraceae & 9 & 2 & 0.067 & 0.556 & 0.016 \\
\hline
\end{tabular}


Table 4 Overrepresented and underespresented plants for each province when compared to the regional plant diversity as recorded in the PNG Plant Database (Continued)

\begin{tabular}{|c|c|c|c|c|c|}
\hline Theaceae & 9 & 2 & 0.067 & 0.556 & 0.016 \\
\hline Begoniaceae & 10 & 2 & 0.060 & 0.518 & 0.009 \\
\hline Balsaminaceae & 4 & 1 & 0.053 & 0.716 & 0.002 \\
\hline Caprifoliaceae & 4 & 1 & 0.053 & 0.716 & 0.002 \\
\hline Icacinaceae & 4 & 1 & 0.053 & 0.716 & 0.002 \\
\hline Oxalidaceae & 4 & 1 & 0.053 & 0.716 & 0.002 \\
\hline Selaginellaceae & 4 & 1 & 0.053 & 0.716 & 0.002 \\
\hline Usneaceae & 4 & 1 & 0.053 & 0.716 & 0.002 \\
\hline \multicolumn{6}{|c|}{ Underrepresented Families } \\
\hline Orchidaceae & 191 & 1 & 0.001 & 0.029 & -0.009 \\
\hline \multicolumn{6}{|c|}{ Bougainville (BV) Reports vs PNG PlantDB (BV Total Flora) } \\
\hline & $\begin{array}{l}\text { \# in PNG } \\
\text { PlantDB }\end{array}$ & $\begin{array}{l}\text { \# in BV Medical Plants } \\
\text { Reports }\end{array}$ & $\begin{array}{l}\text { Inferior Credible } \\
\text { Interval }\end{array}$ & $\begin{array}{l}\text { Superior Credible } \\
\text { Interval }\end{array}$ & $\begin{array}{l}\text { Difference to interval } \\
\text { (margin) }\end{array}$ \\
\hline Total & 1524 & 154 & 0.087 & 0.117 & - \\
\hline \multicolumn{6}{|c|}{ Overrepresented Families } \\
\hline Verbenaceae & 3 & 3 & 0.398 & 0.994 & 0.280 \\
\hline Musaceae & 2 & 2 & 0.292 & 0.992 & 0.175 \\
\hline Zingiberaceae & 19 & 9 & 0.272 & 0.685 & 0.155 \\
\hline Gnetaceae & 3 & 2 & 0.194 & 0.932 & 0.077 \\
\hline Arecaceae & 19 & 7 & 0.191 & 0.592 & 0.074 \\
\hline Marattiaceae & 6 & 3 & 0.184 & 0.816 & 0.067 \\
\hline Caricaceae & 1 & 1 & 0.158 & 0.987 & 0.041 \\
\hline Xanthorrhoeaceae & 1 & 1 & 0.158 & 0.987 & 0.041 \\
\hline Leeaceae & 4 & 2 & 0.147 & 0.853 & 0.029 \\
\hline Fabaceae & 53 & 12 & 0.135 & 0.356 & 0.018 \\
\hline Thelypteridaceae & 9 & 3 & 0.122 & 0.652 & 0.004 \\
\hline Malvaceae & 30 & 7 & 0.119 & 0.411 & 0.001 \\
\hline \multicolumn{6}{|c|}{ Underrepresented Families } \\
\hline Orchidaceae & 74 & 1 & 0.003 & 0.072 & -0.015 \\
\hline
\end{tabular}

BS Bougainville, EH Eastern Highlands, ES East Sepik, PNG PlantDB Papaua New Guinea Plant Database [10]

medicinal use. No scientific background information was located, hence this particular plant may be understudied. The same is also true for Macaranga darbyshirei Airy Shaw, used in the East Sepik as an antivenom, but not elsewhere mentioned for medicinal purposes.

Pandanus dubius Spreng. was not found to have any properly referenced medicinal annotations, but appears to have a fairly recent research record including discovery of two novel alkaloids, dubiusamines-A and dubiusamines-B [23].

Piper mestonii F.M. Bailey leaves used for fresh cuts and wounds do not seem to be described elsewhere. No biochemical investigation could be located in the Dictionary of Natural Products [24].
Planchonia papuana R. Knuth appears to be not used medicinally elsewhere. It is a timber tree and perhaps as such has not attracted attention; however, in an antiviral screen in our lab fractions from $P$. papuana exhibited anti-HIV activity [25].

Plectranthus parviflorus Willd., along with Plectranthus blumei (Benth). Launert, and Plectranthus myrianthus Briq. belong to a genus prominent for production of essentials oils [26] and with multiple annotations for antimicrobial activity, but do not seem to be described elsewhere in the PNG plant literature. The utilization of these plants for sores, ulcers and fresh cuts appear to be in line with the activities of chemicals found in Plectranthrus species [27]. 
Table 5 Overrepresented and underespresented plants for each province when compared to the regional plant diversity as recorded in the UPNG Traditional Medicines Database

\begin{tabular}{|c|c|c|c|c|c|}
\hline \multicolumn{6}{|c|}{ East Sepik (ES Reports vs UPNG TradMed DB } \\
\hline & $\begin{array}{l}\text { \# in UPNG TradMed } \\
\text { DB }\end{array}$ & $\begin{array}{l}\text { \# in ES Medical Plants } \\
\text { Reports }\end{array}$ & $\begin{array}{l}\text { Inferior Credible } \\
\text { Interval }\end{array}$ & $\begin{array}{l}\text { Superior Credible } \\
\text { Interval }\end{array}$ & $\begin{array}{l}\text { Difference to interval } \\
\text { (margin) }\end{array}$ \\
\hline Total & 1176 & 203 & 0.152 & 0.195 & - \\
\hline \multicolumn{6}{|c|}{ Overrepresented Families } \\
\hline Convolvulaceae & 6 & 5 & 0.421 & 0.963 & 0.226 \\
\hline Arecaceae & 10 & 7 & 0.390 & 0.891 & 0.195 \\
\hline Marantaceae & 2 & 2 & 0.292 & 0.992 & 0.097 \\
\hline Apocynaceae & 25 & 9 & 0.202 & 0.557 & 0.007 \\
\hline \multicolumn{6}{|c|}{ Underrepresented Families } \\
\hline Verbenaceae & 22 & 0 & 0.001 & 0.148 & -0.004 \\
\hline \multicolumn{6}{|c|}{ Eastern Highlands (EH) vs UPNG TradMed DB } \\
\hline & $\begin{array}{l}\text { \# in UPNG TradMed } \\
\text { DB }\end{array}$ & $\begin{array}{l}\text { \# in EH Medical Plants } \\
\text { Reports }\end{array}$ & $\begin{array}{l}\text { Inferior Credible } \\
\text { Interval }\end{array}$ & $\begin{array}{l}\text { Superior Credible } \\
\text { Interval }\end{array}$ & $\begin{array}{l}\text { Difference to interval } \\
\text { (margin) }\end{array}$ \\
\hline Total & 1176 & 147 & 0.107 & 0.145 & - \\
\hline \multicolumn{6}{|c|}{ Overrepresented Families } \\
\hline Monimiaceae & 2 & 2 & 0.292 & 0.992 & 0.147 \\
\hline Plantaginaceae & 2 & 2 & 0.292 & 0.992 & 0.147 \\
\hline Winteraceae & 2 & 2 & 0.292 & 0.992 & 0.147 \\
\hline Melastomataceae & 7 & 4 & 0.245 & 0.843 & 0.100 \\
\hline Asparagaceae & 5 & 3 & 0.223 & 0.882 & 0.078 \\
\hline Smilacaceae & 5 & 3 & 0.223 & 0.882 & 0.078 \\
\hline Onagraceae & 3 & 2 & 0.194 & 0.932 & 0.049 \\
\hline Pittosporaceae & 3 & 2 & 0.194 & 0.932 & 0.049 \\
\hline Asteraceae & 47 & 13 & 0.170 & 0.418 & 0.024 \\
\hline Phyllanthaceae & 1 & 1 & 0.158 & 0.987 & 0.013 \\
\hline Caryophyllaceae & 1 & 1 & 0.158 & 0.987 & 0.013 \\
\hline Chloranthoceae & 1 & 1 & 0.158 & 0.987 & 0.013 \\
\hline Elaegnaceae & 1 & 1 & 0.158 & 0.987 & 0.013 \\
\hline Oleaceae & 1 & 1 & 0.158 & 0.987 & 0.013 \\
\hline Polygalaceae & 1 & 1 & 0.158 & 0.987 & 0.013 \\
\hline Tiliaceae & 1 & 1 & 0.158 & 0.987 & 0.013 \\
\hline Proteaceae & 4 & 2 & 0.147 & 0.853 & 0.001 \\
\hline \multicolumn{6}{|c|}{ Underrepresented Families } \\
\hline Euphorbiaceae & 88 & 3 & 0.012 & 0.095 & -0.012 \\
\hline
\end{tabular}

Bougainville (BV) Reports vs UPNG TradMedDB

$\begin{array}{llllll} & \begin{array}{l}\text { \# in UPNG TradMed } \\ \text { DB }\end{array} & \begin{array}{l}\text { \# in BV Medical Plants } \\ \text { Reports }\end{array} & \begin{array}{l}\text { Inferior Credible } \\ \text { Interval }\end{array} & \begin{array}{l}\text { Superior Credible } \\ \text { Interval }\end{array} & \begin{array}{l}\text { Difference to interval } \\ \text { (margin) }\end{array} \\ \text { Total } & 1177 & 146 & 0.106 & 0.144 & \text { meh } \\ \text { Overrepresented Families } & & & 0.390 & 0.891 & 0.246 \\ \text { Arecaceae } & 10 & 7 & 0.292 & 0.992 & 0.148 \\ \text { Leeaceae } & 2 & 2 & & \end{array}$


Table 5 Overrepresented and underespresented plants for each province when compared to the regional plant diversity as recorded in the UPNG Traditional Medicines Database (Continued)

\begin{tabular}{clllll}
\hline & 2 & 2 & 0.292 & 0.992 & 0.148 \\
Rhizophoraceae & & & & & 0.882 \\
Thelypteridaceae & 5 & 3 & 0.223 & 0.594 & 0.079 \\
Zingiberaceae & 23 & 9 & 0.221 & 0.643 & 0.071 \\
Malvaceae & 17 & 7 & 0.215 & 0.987 & 0.014 \\
Salicaceae & 1 & 1 & 0.158 & 0.987 & 0.014 \\
Pteridaceae & 1 & 1 & 0.158 & 0.987 & 0.014 \\
Scrophulariaceae & 1 & 1 & 0.158 & 0.755 & 0.013 \\
Marattiaceae & 7 & 3 & 0.157 & 0.421 & 0.006 \\
Moraceae & 38 & 10 & 0.150 & 0.853 & 0.003 \\
Gnetaceae & 4 & 2 & 0.147 & & \\
Underrepresented Families & & &
\end{tabular}

Riedelia corallina (K. Schum.) Valeton, in the Zingiber family, is used for menstrual cramps, but seems to be otherwise undescribed for medicinal uses elsewhere.

The leaves of Schismatoglottis calyptrata (Roxb.) Zoll. \& Moritzi are used in East Sepik to treat skin sores. No other mention was found in the PNG literature. The stems of Schismatoglottis calyptrata (Roxb.) Zoll. \& Moritzi are however used in Chinese medicine for treatment of lumbago and arthralgia [18].

Sterculia shillinglawii F. Muell. has no previous annotation for PNG, but is known to be used in the Solomon island as a tonic and to reduce fever [18].

Tinospora arfakiana Becc. likewise lacks further medicinal descriptions from PNG and does not seem to have been studied from any other area, making it a potentially understudied plant.

Uncaria lanosa var. appendiculata (Benth.) Ridsdale was mentioned twice in the reports and in both instances to treat fever, but also gastrointestinal diseases, malaria, and malnutrition. No other mention for ethnomedical use could be located from PNG or other locales. However, a recent publication hints at a potential anti-depressant effect of ethanolic extracts of Uncaria lanosa var. appendiculata (Benth.) Ridsdale [28].

Uncaria orientalis Guillaumin, used to treat shortness of breath in the East Sepik, lacks pharmacological annotation, but has been investigated extensively biochemically $[29,30]$.

\section{Conclusions}

This report shows that in the East Sepik province of PNG the patterns of plant usage for medicinal indications is highly varied. This is true even though many of the same plants are used in ethnologically distinct regions. There is a tendancy for widely used plants to be used for multiple diseases, often with differing preparation of the parts utilized and differing modes of administration. One such example is Alstonia scholaris (L.) R.Br. which shares only the route of administration between all areas. Regardless, plants not previously documented as being used medicinally can still be uncovered, e.g., Cascabela thevetia (L.) Lippold, a plant known to contain highly toxic cardiac glycosides [31] and Dendrocnide cordata (Warb. ex H.J.P. Winkl.) Chew cannot be found as being used medicinally, however, toxicity from leaves, which are used in East Sepik, has been documented [18].

Comparison of plant utilization across study areas can likewise uncover plants which share use. A good example is the genus Alpinia, for which gasteroenterological, respiratory and reproductive use are cited for Bougainville. In the Eastern Highlands it is used for gasteroenterological and respiratory conditions. In the East Sepik it is also usedfor respiratory conditions. Alpinia is in the ginger family, widely used culinarily and medicinally around the world, with traditional medicinal uses for several of the described symptoms.

Likewise, dissemination of knowledge of useful phytomedicinal practices amongst areas that share key flora may aid health practices in those areas. In any case, further studies and phytochemical analyses need to be completed before addition of plants to the pharmacopeia for PNG (a goal of the National Policy for Traditional Medicne in PNG). The UPNG Traditional Medicines Database, while still being populated with data, can already be utilized to show correlations and extract lead information for targeting certain plants for further study. Further enhancements and perhaps adaptation of other data sources (e.g., the PNG Plant Database with up-to-date plant nomenclature) would drive statistical discovery of 
medicinally neglected plant genera. It is shown here that transregional comparisons are possible, but require careful recoding of previous reports and standardization of database entries and terminology.

Analysis of frequency of use of plant families in the medical tradition points to certain biases. This can ultimately be useful in targeting plants for biochemical investigation. However, if the desired outcome of the ethnobotany endeavor is to highlight useful plants for the pharmacopeia, then finer grained data is needed in order to dissect the wealth of information gathered, (e.g. precise geographic location including environmental conditions, etc.). Annotation with biochemical information, conservation status, toxicity data would yield utility for a more diverse set of scientists. To this end the diverse efforts of PNG botany, ethnobotany, ethnopharmacology and plant conservation need to collaborate more rigorously to define useful interfaces for each other's data needs. Nevertheless, we have been able to successfully show that medicinal plant use in terms of families utilized in the East Sepik resembles Bougainville provinces more than it does the Eastern Highlands. Future work with larger data sets will address whether such similarities are due to similarities of available flora or other causes.

\section{Competing interests}

The authors declare that they have no competing interests.

\section{Authors' contributions}

MK wrote the manuscript and prepared statistical data and analysis. LRB wrote and edited the manuscript. KMB prepared ethnobotanical data sets for use in the manuscript. DAK, GW, BK, and MS performed interviews and wrote reports under guidance of PPR and TM who also reviewd the manuscript. All authors read and approved the final manuscript.

\section{Acknowledgements}

The reports compiled in this manuscript were "The Traditional Medicinal Plants and Practices in the Waskuk Hills Area of Ambunti District in the East Sepik Province"by Dickson Andrew Kehop (2005), "Ethnobotanical Survey of Traditional Medicine in the East Yangoru, East Sepik Province, Papua New Guinea" by Graham Wavimbukie (2004), "Traditional Medicine Practices in Niungweko: I and Kunjingini (Mul) Areas of the Wosera-Gawi District in East Sepik" by Boniface Kinminja (2006) and "Traditional Medicinal Plants and Practices in Kairiru Island East Sepik Province Papua New Guinea" by Malcom Sabak (2004) Many locally recognized helears contributed to this work: Isaac Hames, Lamech Mesung, Joshua Matambopi, Lillian Nenipa, Evelyn John, Rebecca Waikola, Zippora Gamba and John Wekuai (DK), Ms. Yakupa, Stanley Wingewi, Chris Tapukata, Doughlas Yandu, Adam Knokrakundi, Bill Saun, Ambrose Ganjal, Mr. Yakop and Mr. Paliak (BK), Simon Parimie, Lawrence Parimie, Arnold Sauseha, Freddy Kavi, Nancy Kavi, Camillus Waranaka, Columba Waranaka, Wally Nangusimbie, Andrew Hipanare, Alphonsa Humunda, Paul Hahiva, Jacob Kwale John Pavuhau, Agnes Niniehau and John Nimiehau (GW), Wow B. Woyeng, Wow J. Samiek, Wow P. Wilbarau, Wow Wapsi, Wow Kaisem, Brata Rammy N., Wow Sareo, Wow Takswau, Mr. and Mrs Tambuna August, Wow Temos, Ms. Vero T. and Ms. Sumin K (MS). We also acknowledge UPNG and FRI Herbarium staff for assistance in identification of medicinal plant vouchers. This work was funded by US NIH support through the Fogarty International Center, ICBG 5U01T006671, and the Wheeler Foundation through Wells Fargo, UT. Dr. Rai, Dr. Matainaho and students had additional support from by the National Department of Health, Papua New Guinea and the University of Papua New Guinea.

\section{Author details}

${ }^{1}$ Center for Biopharmaceutical Research, De La Salle Health Sciences Institute, Dasmarinas, Cavite, Philippines. ${ }^{2}$ School of Medicine and Health Sciences, University of Papua New Guinea, PO Box 5623, Boroko, NCD, Papua New Guinea. ${ }^{3}$ Department of Pharmacology and Toxicology, University of Utah, 30 S. 2000 E., Salt Lake City 84112 UT, USA.

Received: 2 June 2015 Accepted: 24 October 2015

Published online: 14 November 2015

\section{References}

1. Asher RE. The encyclopedia of language and linguistics. Oxford: Pergamon Press; 1994.

2. Ethnologue. Grimes B. SIL International; Dallas: 2000. https:// www.ethnologue.com/. Accessed October 2015

3. Summerhayes GR, Leavesley M, Fairbairn A, Mandui H, Field J, Anne Ford A, et al. Human adaptation and plant use in highland New Guinea 49,000 to 44,000 years ago. Science. 2010;330:78-81.

4. PNG district and provincial profiles. Available from: http://docslide.us/ documents/png-profiles-report-140410.html. Accessed October 2015.

5. National Department of Health. National policy on traditional medicine. Waigani: National Department of Health; 2007.

6. Ministry of Health. National health plan 2001-2010 volume I. Port Moresby: Ministry of Health; 2000.

7. Rai PP. Traditional medicine in Papua New Guinea. National Capital District: University of Papua New Guinea Printery; 2004.

8. Waruruai J, Sipana B, Koch M, Barrows LR, Matainaho TK, Rai PP. An ethnobotanical survey of medicinal plants used in the Siwai and Buin Districts of the Autonomous Region of Bougainville. J Ethnopharm. 2011;138:564-77.

9. Jorim RY, Korape S, Legu W, Koch M, Barrows LR, Matainaho TK, et al. An ethnobotanical survey of medicinal plants used in the Eastern Highlands of Papua New Guinea. J Ethnobiol Ethnomed. 2012;8:47.

10. Conn BJ, Lee LL, Kiapranis, R. PNGPlants database. [http://www.pngplants.org/] Accessed May 2014.

11. Weckerle CS, Cabras S, Castellanos ME, Leonti M. Quantitative methods in ethnobotany and ethnopharmacology: considering the overall flora Hypothesis testing for over- and underused plant families with the Bayesian approach. J Ethnopharm. 2011;137:837-43.

12. Prescott TA, Kiapranis R, Maciver SK. Comparative ethnobotany and in-thefield antibacterial testing of medicinal plants used by the Bulu and inland Kaulong of Papua New Guinea. J Ethnopharmacol. 2012;139:497-503.

13. Lansky EP, Paavilainen HM. Figs: the genus Ficus (Traditional Herbal Medicines for Modern Times). Boca Raton: CRC Press; 2010.

14. Boyce PC. A review of Alocasia (Araceae: Colocasieae) for Thailand including a novel species and new species records from South-West Thailand. Thai For Bull. 2008;36:1-17.

15. Peng Q, Cai H, Sun X, Li X, Mo Z, Shi J. Alocasia cucullata exhibits strong antitumor effect in vivo by activating antitumor immunity. PLoS One. 2013;8:e75328.

16. Kaur A, Kamboj SS, Singh J, Saxena AK, Dhuna V. Isolation of a novel N-acetylD-lactosamine specific lectin from Alocasia cucullata (Schott.). Biotechnol Lett. 2005;272:1815-20.

17. STUARTXCHANGE. http://www.stuartxchange.com/ Accessed Jan 2015 trough March 2015.

18. Johnson T. CRC ethnobotany desk reference. Boca Raton: CRC Press LLC; 1998.

19. Folkers K, Unna K. Erythrina alkaloids. V. Comparative curare-like potencies of species of the genus Erythrina. J Am Pharm Assoc. 1939;28:1019-28.

20. Bourdy G, Walter A. Maternity and medicinal plants in Vanuatu I. The cycle of reproduction. J Ethnopharm. 1992;37:179-96.

21. Bourdy G, Walter A. Maternity and medicinal plants in Vanuatu I. The cycle of reproduction. J Ethnopharm. 1992;37:179-96.

22. Dr. Duke's phytochemical and ethnobotanical databases. http://www.ars-grin.gov/cgi-bin/duke/ethnobot.pl Accessed September 2014.

23. Tana MA, Kitajimaa M, Kogurea N, Nonatob MG, Takayama H. Isolation and total syntheses of two new alkaloids, dubiusamines- $A$, and -B, from Pandanus dubius. Tetrahedron. 2010;66:3353-9.

24. Dictionary of natural products. Available at http://dnp.chemnetbase.com). Accessed September 2014. 
25. Barrows LR, Matainaho TK, Ireland CM, Miller S, Carter GT, Bugni T, et al. Making the most of Papua New Guinea's biodiversity: establishment of an integrated set of programs that link botanical survey with pharmacological assessment in "The Land of the Unexpected". Pharmaceutical Biol. 2009;47:795-808

26. Williams C. Medicinal plants in Australia volume 4: an Antipodean apothecary. Kenthurst: Rosenberg Publishing; 2013.

27. Lukhoba CW, Simmonds MSJ, Paton A. Plectranthus: a review of ethnobotanical uses. J Ethnopharmacol. 2006;103:1-24.

28. Hsu LC, Ko YJ, Cheng HY, Chang CW, Lin YC, Cheng YH, et al. Antidepressant-like activity of the ethanolic extract from Uncaria lanosa Wallich var. appendiculata Ridsd in the forced swimming test and in the tail suspension test in mice. Evid Based Complement Alternat Med. 2012;2012:497302. doi:10.1155/2012/497302. Epub 2012 Apr 9.

29. Croquelois G, Miet C, Poisson J, Sevenet T. Oxyindole alkaloids of Uncaria orientalis Guill. (Rubiaceae). Ann Pharm Fr. 1977;35:417-8.

30. Phillipson JD, Hemingway SR. Alkaloids of Uncaria attenuata, U. orientalis and U. Canescens. Phytochemistry. 1975;14:1855-63.

31. Langford SD, Boor PJ. Oleander toxicity: an examination of human and animal toxic exposures. Toxicology. 1996;109:1-13.

32. Holdsworth D. Medicinal plants of the Morobe Province, Papua New Guinea. Part V. The Upper Watut. Int J Crude Drug Res. 1987;25:225-30.

33. Haberle S. Ethnobotany of the Tari Basin, Southern Highlands Province, Papua New Guinea. Canberra: Biogeography \& Geomorphology Department; 2005.

34. Holdsworth DK. Medicinal plants of Papua New Guinea. Noumea: South Pacific Commission; 1977. Technical Paper No. 175.

35. Hide R, Kimin M, Kora A, Kua G, Kua K. A checklist of some plants in the territory of the Sinasina Nami (Simbu Province, Papua New Guinea), with notes on their uses. Working papers in anthropology, archaeology, linguistics and Maori Studies No. 54. Auckland: Dept. Anthropology, U. Auk. Private Bag; 1979.

36. Holdsworth D, Rali T. A survey of medicinal plants of the Southern Highlands, Papua New Guinea. Int J Crude Drug Res. 1989;27:1-8.

37. Holdsworth D, Lacanienta E. Traditional medicinal plants of the Central Province of Papua New Guinea. Part II. Int J Crude Drug Res. 1981;19:155-67.

38. Holdsworth DK, Farnworth ER. A phytochemical survey of medicinal and poisonous plants of the Central District of Papua. Sci New Guinea. 1974;2:155-63.

39. Goeltenboth F, Holdsworth DK, Sakulas H, Thredgold H, Woodley E. Medicinal plants in Papua New Guinea Part I: Morobe Province. E. Woodley (Ed.). Weikersheim, Germany: Verlag Josef Margraf publiser; 1991.

40. Holdsworth D. Traditional medicinal plants used in the treatment of gastric ailments. PNG Med J. 1978;21:175-83.

41. Johannes A. Medicinal plants of the Nekematigi of the Eastern Highlands of New Guinea. Econ Bot. 1975;29:268-77.

42. Rai PP, Matainaho T, Saulei S, Ambihaipahar U. Medicinal Plants in Papua New Guinea. Manila: WHO (World Health Organization), Western Pacific Region; 2009.

43. Paijmans K. New Guinea vegetation. Canberra: Australian National University Press; 1976.

44. Holdsworth DK. Some medicinal plants of the Marawaka Kukukuku people. Science in New Guinea. 1973;1:17-20.

45. Holdsworth D. High altitude medicinal plants of Papua New Guinea. Int J Crude Drug Res. 1989;27:95-100.

46. Holdsworth D, Lacanienta E. Traditional medicinal plants of the Central Province of Papua New Guinea. Part I. Int J Crude Drug Res. 1981;19:141-54.

47. Holdsworth DK, Gelege N, Close K, Close A. Some traditional medicina plants of Papua New Guinea. Sci New Guinea. 1975;3:165-72.

48. Holdsworth D. Traditional medicinal. Traditional medicinal plants used in the treatment of malaria and fevers in Papua New Guinea. PNG Med J. 1975;18:142-8.

49. Holdsworth DK. Medicinal plants from Milne Bay, Eastern Papua. Sci New Guinea. 1975;3:98-102.

50. Hide RL. South Simbu: studies in demography, nutrition and subsistence. Research report of the Simbu land use project Vol VI. 1984

51. Henty EE. Harmful plants in Papua New Guinea. Lae: Dept. of Forests; 1980.

52. Holdsworth DK, Tringen SB. Medicinal plants of Sepik. Sci New Guinea. 1973;1:5-10

53. Telban JB. The role of medical ethnobotany in ethnomedicine: a New Guinea example. Ethnobiol. 1988:8:149-69.
54. Stopp K. Medicinal plants of the Mt. Hagen People (Mbowamb) in New Guinea. Econ Bot. 1963;17:16-22.

55. Skingle DC. Some medicinal herbs used by the natives of New Guinea. Mankind. 1970;7:223-5.

56. Rai P. Traditional uses of plants for health and healing in Bougainville Part 1. Madang, Papua New Guinea Traditional Health: HORIZONT3000; 2012.

57. Holdsworth DK. Traditional medicinal plants of the North Solomons Province Papua New Guinea. Int J Crude Drug Res. 1980;18:33-44.

58. Nick A, Rali T, Sticher O. Biological screening of traditional medicinal plants from Papua New Guinea. J Ethnopharm. 1995;49:147-56.

59. Holdsworth DK, Tamanabae R, Small OR, Farnworth E. A phytochemical survey of medicinal and poisonous plants from the Northern District of Papua. Papua New Guin Sci Soc Proc. 1974;25:85-98.

60. Holdsworth D. Traditional medicinal plants of Central Province PNG. Part IV The Goilala mountain people. Int J Crude Drug Res. 1987;25:231-5.

61. Holdsworth DK. A phytochemical survey of medicinal plants of the $D$ 'Entrecasteaux Islands, Papua. Sci New Guinea. 1974;1:164-71.

62. Holdsworth DK, Hurley C, Rayner SE. Traditional medicinal plants of New Ireland Papua New Guinea. Pharm Biol. 1980;18:131-9.

63. Holdsworth DK, Balun L. Medicinal plants of the East and West Sepik provinces, Papua New Guinea. Int J Pharmacog. 1992;30:218-22.

64. Holdsworth D, Wamoi B. Medicinal plants of the Admiralty Islands, Papua, New Guinea. Part 1. Int J Crude Drug Res. 1982;20:169-81.

65. Holdswort DK, Balun L. Ethnomedicine of the gulf province of Papua New Guinea Part I: The mountains around Kanabea and Kaintiba. Int J Crude Drug Res. 1988;26:51-5.

66. Blackwood B. Use of plants among the Kukukuku of Southeast-Central New Guinea. Proc Sixth Pac Sci Congress Pac Sci Assoc. 1939;4:111-26.

67. Holdsworth DK, Damas K. Medicinal plants of Morobe Province, Papua New Guinea. III. The Finschhafen coast. Int J Crude Drug Res. 1986;24:217-25.

68. Holdsworth DK. Phytomedicine of the Gazelle Peninsula, New Britain. Sci New Guinea. 1975;3:32-40.

69. Holdsworth D, Kerenga K. Medicinal plants of the Western Highlands, Papua New Guinea New Guinea. Int J Crude Drug Res. 1987;25:171-6.

70. Blackwood B. Treatment of the sick in the Solomon Islands. Folklore. 1935;46:148-61.

71. Sillitoe P. An ethnobotanical account of the plant resources of the Wola region, Southern Highlands Province. Papua New Guinea J Ethnobiol. 1995;15:201-35.

72. Holdsworth D, Giheno J. A preliminary survey of highland medicinal plants. Sci New Guinea. 1975;3:191-8.

73. Holdsworth D. Traditional medicinal plants of Central Province PNG. Part III Int J Crude Drug Res. 1987;25:103-12.

74. Holdsworth D, Kerenga KA. A survey of medicinal plants in the Simbu Province, Papua New Guinea. Int J Crude Drug Res. 1987;25:183-7.

75. Holdsworth D, Sakulas H. High altitude medicinal plants of Papua New Guinea. Part II, Mount Wilhelm, Simbu Province. Int J Pharmacog. 1992;30:1-4.

76. Holdsworth DK, Longley RP. Some medicinal and poisonous plants from the Southern Highlands District of Papua. Proc Papua New Guinea Scient Soc. 1972;24:21-4.

77. Holdsworth D, Sakulas H. Medicinal plants of the Morobe Province Part II. The Aseki Valley. Int J Crude Drug Res. 1986;24:31-40.

78. Holdsworth DK, Heers G. Some medicinal and poisonous plants from the Trobriand Islands. Rec Papua New Guinea Mus. 1971;1:37-40.

\section{Submit your next manuscript to BioMed Central and take full advantage of:}

- Convenient online submission

- Thorough peer review

- No space constraints or color figure charges

- Immediate publication on acceptance

- Inclusion in PubMed, CAS, Scopus and Google Scholar

- Research which is freely available for redistribution 